\title{
Sum Rule Measurements of the Spin-Dependent Compton Amplitude (Nucleon Spin Structure at $Q^{2}=0$ ) - ILEGS EXPS. III/LIS -
}

\section{LEGS-Spin Collaboration}
D. Babusci ${ }^{2}$, H. Baghaei ${ }^{14}$, M. Blecher ${ }^{13}$, M. Breuer ${ }^{9}$, A. Caracappa ${ }^{1}$, A. Cichocki14,
C. Commeaux ${ }^{9}$, J.-P. Didelez ${ }^{9}$, Q. Fan ${ }^{12}$, G. Giordano ${ }^{2}$, V. Gladyshev ${ }^{14}$, K. Hicks ${ }^{8}$, S. Hoblit ${ }^{1,14}$, P. Hoffmann-Rothe ${ }^{9}$, A. Honig12, O. C. Kistner ${ }^{1}$, M. Khandaker ${ }^{7}$, Z. Li ${ }^{6}$, R. Lindgren 14 , M. Lucas ${ }^{11}$, G. Matone ${ }^{2}$, L. Miceli 111 , B. Norum ${ }^{14}$, B. M. Preedom ${ }^{11}$, M. Rigney ${ }^{9}$, A. M. Sandorfi ${ }^{1}$, M. Sanzoni ${ }^{3}$, C. Schaerf ${ }^{10}$, R. Sealock ${ }^{14}$, C. Smith ${ }^{14}$, H. Ströher ${ }^{4}$, C. E. Thorn ${ }^{1}$, X. Wei ${ }^{12}$, C.R. Wuest ${ }^{5}, K$. Wang ${ }^{14}$ and C. S. Whisnant ${ }^{11}$

\author{
1) Brookhaven National Laboratory, Upton, NY 11973 \\ 2) Laboratori Nationali di Frascati-INFN, Rome, Italy \\ 3) Universita di Genova and INFN-Sezione di Genova, Genova, Italy \\ 4) II Physikalisches Institut, Universität Gießen, Gießen, Germany \\ 5) Lawrence Livermore Laboratory, Livermore, CA 94550 \\ 6) Christopher Newport University and CEBAF, Newport News, VA 23606 \\ 7) Norfolk State University and CEBAF, Norfolk, VA 23504 \\ 8) Ohio University, Athens, $\mathrm{OH} 45701$ \\ 9) IN2P3, IPN Orsay, France \\ 10) Universita di Roma Tor Vergata and INFN-Sezione di Roma 2, Rome, Italy \\ 11) University of South Carolina, Columbia, SC 29208 \\ 12) Syracuse University, Syracuse, NY 13244 \\ 13) Virginia Polytechnic Institute and State University, Blacksburg, VA 24061 \\ 14) University of Virginia, Charlottesville, VA 22901
}

\begin{abstract}
Energy weighted integrals of the difference in helicity-dependent photo-production cross sections $\left(\sigma_{1 / 2}-\sigma_{3 / 2}\right)$ provide information on the nucleon's Spin-dependent Polarizability $(\gamma)$, and on the spin-dependent part of the asymptotic forward Compton amplitude through the Drell-Hearn-Gerasimov (DHG) sum rule. (The latter forms the $\mathrm{Q}^{2}=0$ limit of recent spin-asymmetry experiments in deep-inelastic lepton-scattering.) There are no direct measurements of $\sigma_{1 / 2}$ or $\sigma_{3 / 2}$, for either the proton or the neutron. Estimates from current $\pi$-photo-production multipole analyses, particularly for the protonneutron difference, are in good agreement with relativistic-1-loop Chiral calculations $(\chi P T)$ for $\gamma$ but predict large deviations from the DHG sum rule. Either (a) both the 2loop corrections to the Spin-Polarizability are large and the existing multipoles are wrong, or (b) modifications to the Drell-Hearn-Gerasimov sum rule are required to fully describe the isospin structure of the nucleon.

The helicity-dependent photo-reaction amplitudes, for both the proton and the neutron, will be measured at LEGS from pion-threshold to $470 \mathrm{MeV}$. In these doublepolarization experiments, circularly polarized photons from LEGS will be used with SPHICE, a new frozen-spin target consisting of $\vec{H} \cdot \vec{D}$ in the solid phase. Reaction channels will be identified in SASY, a large detector array covering about $80 \%$ of $4 \pi$. A high degree of symmetry in both target and detector will be used to minimize systematic uncertainties.
\end{abstract}




\section{Contents}

Scientific Motivation:

The Spin -Dependent Compton Amplitude and Nucleon Spin Structure

The Spin-Dependent Polarizability of the Nucleon

The Drell-Hearn-Gerasimov Sum Rule

Connections with Deep Inelastic Scattering

Measurements on the proton

Comparisons of the Neutron and Proton

The Role of the $\Delta$ (1232) Resonance

Estimates from Photoproduction Multipoles

Consequences of the Incompatibility of Predictions for the Proton-Neutron Difference

SPHICE:

A Strongly Polarized $\mathbb{H}$ ydrogen and Deuterium ICEE Target

Hydrogen polarization by High-Field Relaxation with Ortho- $\mathrm{H}_{2}$ Doping

Deuteron polarization by Adiabatic Fast Passage

SPHICE fabrication and operation

Advantages over conventional targets

LEGS:

Energy Range of Measurements with Circularly Polarized Beams

Detector Array:

Measurements of the Polarized Cross Sections

SASY - The Spin ASYmmetry Detector Array

Monte Carlo Simulations

Count rate Estimates

References

Appendix:

Technical Review, June 7, 1994

Report of the Technical Review Panel on SPHICE

- the HD Polarized Target for LEGS

BNL Action on Recommendations of the June 7, 1994 Technical Review 


\section{DISCLAIMER}

This report was prepared as an account of work sponsored by an agency of the United States Government. Neither the United States Government nor any agency thereof, nor any of their employees, make any warranty, express or implied, or assumes any legal liability or responsibility for the accuracy, completeness, or usefulness of any information, apparatus, product, or process disclosed, or represents that its use would not infringe privately owned rights. Reference herein to any specific commercial product, process, or service by trade name, trademark, manufacturer, or otherwise does not necessarily constitute or imply its endorsement, recommendation, or favoring by the United States Government or any agency thereof. The views and opinions of authors expressed herein do not necessarily state or reflect those of the United States Government or any agency thereof. 


\section{DISCLAIMER}

Portions of this document may be illegible in electronic image products. Images are produced from the best available original document. 


\section{Scientific Motivation:}

\section{The Spin-Dependent Compton Amplitude and Nucleon Spin Structure}

The recent experiments on the deep-inelastic scattering of polarized leptons from polarized protons and neutrons have raised interesting questions on the spin-structure of the nucleon(As88,Ad93,An93). Because non-perturbative QCD corrections can potentially influence the interpretation of these measurements, considerable attention has recently been paid to the $Q^{2}$ evolution of the spin observables(AIL89,B193,Bu93,ST93,EK93,Ji93). The $Q^{2}=0$ limit is determined by the total spin-dependent photo-absorption cross sections measured with the photon and nucleon polarizations parallel, $\sigma_{3 / 2}$, and anti-parallel, $\sigma_{1 / 2}$. The combination of the LEGS photon beams, the SPHICE target and the SASY detector array is uniquely well suited to measurements that will make significant impacts on these quantities.

A variety of sum rules have been derived for the integrals of these photo-reaction

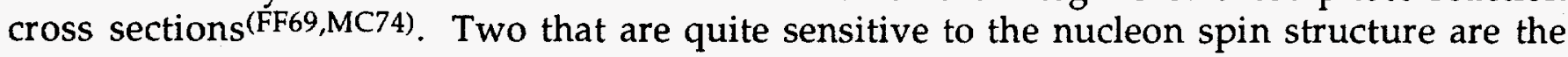
spin-dependent polarizability(BKKM92) (or 'spin polarizability', $\gamma$ ), and the Drell-HearnGerasimov (DHG) integrals (DH66,Ge66). Both are derived from considerations of the forward Compton scattering amplitude of Gell-Mann, Goldberger and Thirring (GGT)(GGT54) which for spin $1 / 2$ targets, using crossing symmetry, takes the form

$$
A(\omega)=f\left(\omega^{2}\right) \vec{\varepsilon}^{\prime} \cdot \vec{\varepsilon}+i \omega g\left(\omega^{2}\right) \vec{\sigma} \cdot\left(\vec{\varepsilon}^{\prime} \times \vec{\varepsilon}\right) .
$$

Here $\vec{\varepsilon}$ and $\vec{\varepsilon}^{\prime}$ are the incident and final photon polarization vectors and $\vec{\sigma}$ is the target spinor. For small values of the photon energy $(\omega)$, the functions $f$ and $g$ can be written as

$$
\begin{aligned}
& f\left(\omega^{2}\right)=f(0)+f^{\prime}(0) \omega^{2}+\mathrm{O}\left(\omega^{4}\right) \\
& g\left(\omega^{2}\right)=g(0)+g^{\prime}(0) \omega^{2}+\mathrm{O}\left(\omega^{4}\right)
\end{aligned}
$$

where the prime-symbol indicates differentiation with respect to $\omega^{2}$. The leading term in the spin-independent amplitude,

$$
f(0)=-\alpha / m \quad,
$$

describes Thomson scattering, the motion of the center-of-charge of the target in response to the electromagnetic field of the photon, and is a simple consequence of gauge invariance. The corresponding spin-dependent term is given by the low-energy theorem of Low, Gell-Mann and Goldberger(Lo54,GG54)

$$
g(0)=-\alpha \kappa^{2} / 2 m^{2},
$$

and appears as a consequence of both gauge and relativistic invariance. 


\section{The Spin-Dependent Polarizability of the Nucleon}

The GGT dispersion relations provide sum rules for the slope parameters of the scattering amplitudes in eqn. (2a), in terms of the total spin-dependent cross sections measured with the photon and nucleon polarizations parallel, $\sigma_{3 / 2}$, and anti-parallel, $\sigma_{y_{2}}$. $f^{\prime}(0)$ is identified with the sum of the electric and magnetic polarizabilities of the target $(\alpha+\beta)^{(\mathrm{Ba60})}$, and by analogy $g^{\prime}(0)$ is referred to as the spin polarizability, $\gamma^{\text {(BKKM92), }}$

and

$$
\alpha+\beta \equiv f^{\prime}(0)=\frac{1}{4 \pi^{2}} \int_{\omega_{0}}^{\infty} \frac{\sigma_{y_{2}}+\sigma_{3 / 2}}{\omega^{2}} d \omega
$$

$$
\gamma \equiv g^{\prime}(0)=\frac{1}{4 \pi^{2}} \int_{\omega_{0}}^{\infty} \frac{\sigma_{y_{2}}-\sigma_{3 / 2}}{\omega^{3}} d \omega
$$

The cross section sum in equation 3 is just the total unpolarized photoabsorption cross section. While the spin dependent effects are masked in this sum, they are manifest in the expression for $\gamma$. The sum rule for $\gamma$ has two very important features. First, the denominator in the integral contains the third power of the photon energy. This means a rapid convergence, limiting the range of energies that must be spanned and a reduction of the experimental uncertainties in its evaluation. Secondly, the polarizabilities are calculable in chiral perturbation theory $(\chi \mathrm{PT})$. In heavy baryon $\chi \mathrm{PT}$ they arise purely from pion loops and so do not involve any unknown parameters. Specifically, Bernard, Kaiser, Kambour and Meißner ${ }^{(B K K M 92)}$ find

and

$$
\alpha_{p}=\alpha_{n}=10 \beta_{p}=10 \beta_{n}=\frac{5 e^{2} g_{A}^{2}}{96 \pi M_{\pi} F_{\pi}^{2}}
$$

$$
\gamma_{p}=\gamma_{n}=\frac{e^{2} g_{A}^{2}}{24 \pi^{2} M_{\pi}^{2} F_{\pi}^{2}}
$$

where $M_{\pi}$ is the charged pion mass, $g_{A}=1.26$ is the axial vector coupling constant, and $F_{\pi}=93.3 \mathrm{MeV}$ is the (weak) pion decay constant.

The polarizabilities $\alpha, \beta$ and $\gamma$ are fundamental properties of nucleon structure which would vanish for point nucleons. There have been several recent experimental. determinations of $\alpha$ and $\beta$. Equation 5 explains the empirical observation that $\alpha_{p, n}>>\beta_{p}, n$ and that $\alpha_{p}+\beta_{p}$ is approximately equal to $\alpha_{n}+\beta_{n}$. Since the numerator of the integral in equation 3 is just the total absorption cross section measured with unpolarized photons, the sum of the spin-independent polarizabilities is fairly well determined. The empirical values for the proton and neutron are $\mathrm{e}^{(\mathrm{DG} 70, \mathrm{Pe} 81)}$

$$
\begin{aligned}
& \alpha_{p}+\beta_{p}=(14.2 \pm 0.3) \times 10^{-4} \mathrm{fm}^{3} \\
& \alpha_{n}+\beta_{n}=(15.8 \pm 0.5) \times 10^{-4} \mathrm{fm}^{3}
\end{aligned}
$$


These compare very well with the value of $14 \times 10^{-4} \mathrm{fm}^{3}$ obtained from equation 5 . Equation 6 predicts a value of $437 \times 10^{-6} \mathrm{fm}^{4}$ for the nucleon spin-dependent polarizability. As yet there have been no direct verifications of this prediction for this fundamental nucleon property.

\section{The Drell-Hearn-Gerasimov Sum Rule}

In 1966, Drell and Hearn(DH66), and Gerasimov(Ge66) independently, combined another GGT dispersion relation,

$$
g(0)-g(\infty)=\frac{1}{4 \pi^{2}} \int_{\omega_{0}}^{\infty} \frac{\sigma_{1 / 2}-\sigma_{3 / 2}}{\omega} d \omega
$$

with the (LGG) low-energy theorem of eqn. (2c), to obtain,

$$
D H G=\int_{\omega_{0}}^{\infty} \frac{\sigma_{1 / 2}-\sigma_{3 / 2}}{\omega} d \omega=-\frac{2 \pi^{2} \alpha}{m^{2}} \kappa^{2},
$$

under the additional assumption that $g(\infty)=0$. This sum rule relates the anomalous magnetic moment of the nucleon, $\kappa$, to the difference between the total cross sections measured with the nucleon spin parallel and anti-parallel to the photons, integrated from the pion production threshold to infinity. Thus, an evaluation of the DHG sum rule requires the measurement of the same helicity components as the spin-dependent polarizability.

In writing eqns. (4) and (8), both integrals are implicitly assumed to converge. This is a reasonably safe assumption for $\gamma$, because of the $1 / \omega^{3}$ weighting in the integrand. However, the lower power in the energy weighting of the DHG integrand requires that the cross section difference, which is just the imaginary part of $g(\omega)$ from the optical theorem, falls off with energy faster than $1 / \ln \omega$. This, and the explicit requirement that $g(\infty)=0$, make the DHG sum rule critically dependent upon high-energy behavior.

\section{Connections with Deep Inelastic Scattering}

By considering the forward scattering of circularly polarized virtual photons, a sum rule for the polarized nucleon structure function, $g_{1}$, can be constructed for $\mathrm{Q}^{2}>0$. The quantity of interest is

$$
\Gamma_{1}\left(Q^{2}\right)=\int_{0}^{1} g_{1}\left(x, Q^{2}\right) d x
$$

where the Björken scaling parameter, $x=Q^{2} / 2 M \omega$, is the integration variable. 


\section{Measurements on the proton:}

The EMC collaboration(As88), scattering polarized muons from polarized protons, reported values for this integral, as

$$
\begin{aligned}
\Gamma_{1}^{p}\left(\overline{Q^{2}}\right) & =0.130 \pm 0.015 \pm 0.018 & & \overline{Q^{2}}=4.8 \mathrm{GeV}^{2} \\
& =0.114 \pm 0.021 \pm 0.019 & & \overline{Q^{2}}=17.2 \mathrm{GeV}^{2}
\end{aligned}
$$

To explore the connection of this integral with the DHG sum rule, Anselmino, Ioffe and Leader(AIL89) consider the following function

$$
I_{1}^{p}\left(Q^{2}\right)=2 \frac{m_{p}^{2}}{Q^{2}} \Gamma_{1}^{p}\left(Q^{2}\right)
$$

which, in the scaling region, becomes increasingly positive as $\mathrm{Q}^{2}$ decreases. Equation 11 can be rewritten as

$$
I_{1}^{P}\left(Q^{2}\right)=\frac{M^{2}}{8 \pi^{2} \alpha} \int_{Q^{2} / 2 M}^{\infty}\left[\frac{\sigma_{y_{2}}\left(\omega, Q^{2}\right)-\sigma_{3 / 2}\left(\omega, Q^{2}\right)}{\omega}\right] d \omega
$$

As $Q^{2} \rightarrow 0, I_{1}^{p}$ takes the form ${ }^{(j i 93)}$

$$
I_{1}^{p}\left(Q^{2} \rightarrow 0\right) \rightarrow \frac{M^{2}}{Q^{2}} F_{1}\left(Q^{2}\right)\left\{F_{1}\left(Q^{2}\right)+F_{2}\left(Q^{2}\right)\right\}-\frac{1}{4} \kappa_{p}^{2} .
$$

Here, the first term comes from virtual Compton scattering, with $F_{1}\left(Q^{2}\right)$ and $F_{2}\left(Q^{2}\right)$ being the Dirac (charge) and Pauli (magnetic moment) form factors of the nucleon. These are included because the EMC measurements (along with the more recent SMC and E142/E143 experiments) made no attempt to separate forward elactic scattering from inelastic processes. The form of the second term assumes the validity of the DHG sum rule in order to represents the inelastic contributions as simply $-1 / 4 \kappa_{p}^{2}$. Although the elastic term is large for small values of $Q^{2}$, momentum conservation requires that it vanish at exactly $\mathrm{Q}^{2}=0$, so that at the real photon point $I_{1}^{p}$ is determined entirely by the DHG integral,

$$
I_{1}^{p}\left(Q^{2}=0\right)=-\frac{1}{4} \kappa_{p}^{2}=-0.804
$$

To join the small positive EMC results with the large negative value at $Q^{2}=0$, there must be an incredibly rapid change in slope at small $Q^{2}$. This rapid slope change has potentially significant ramifications on the interpretation of the EMC measurements. The results of Eq. (10) contradict the Ellis-Jaffe sum rule prediction, $0.189 \pm 0.005$ in the high $\mathrm{Q}^{2}$ limit, and have lead to the conclusion that only a fraction of the proton's spin is carried by the constituent (s-wave) $\mathrm{u}$ and $\mathrm{d}$ quarks. However, if the large changes in $I_{1}^{p}$ that are demanded by eq. (13) are caused, even in part, by non-perturbative corrections 
(higher "twist" effects, which should arise at small values of $\mathrm{Q}^{2}$ but are very difficult to calculate), then such corrections may still be important at the finite $\mathrm{Q}^{2}$ of the EMC experiments and would certainly affect their interpretation. These large changes in $I_{1}^{p}$ are of considerable importance and have recently received a good deal of attention(AIL89,B193, Bu93,ST93,EK93,Ji93).

\section{Comparisons of the Neutron and Proton:}

A potentially less model dependent test of the nucleon spin structure comes from a comparison between the proton and neutron. Assuming only chiral U(6) $\times \mathrm{U}(6)$ current algebra, Björken obtained sum rules for the spin structure functions of the proton and neutron ${ }^{(B j 66)}$ at high $Q^{2}$. The difference between the proton and neutron structure functions is related to the vector and axial vector $\beta$-decay coupling constants, which with QCD corrections up to twist- 4 , is

$$
\Gamma_{1}^{p-n}\left(Q^{2}\right)=\int_{0}^{1}\left[g_{1}^{p}\left(x, Q^{2}\right)-g_{1}^{n}\left(x, Q^{2}\right)\right] d x=\frac{1}{6} \frac{g_{A}}{g_{V}}\left(1-\frac{\alpha_{s}\left(Q^{2}\right)}{\pi}\right)
$$

This difference in structure functions can be related to the difference between the DHG sum rules for the proton and neutron by writing, as before

$$
I_{1}^{p-n}\left(Q^{2}\right)=\frac{2 M^{2}}{Q^{2}} \Gamma_{1}^{p-n}\left(Q^{2}\right)
$$

Then at $Q^{2}=0$, assuming the validity of the DHG sum rule for the inelastic contributions from both the proton and neutron in eq. (13), this becomes

$$
I_{1}^{p-n}\left(Q^{2}=0\right)=1 / 4\left(\kappa_{n}^{2}-\kappa_{p}^{2}\right)=+0.112 \quad .
$$

Ellis and Karliner ${ }^{(\mathrm{EK} 93)}$ have made a re-analysis of the EMC(As88) measurements and combined it with the SMC(Ad93), E142(An93) and NMC(Am92) data to obtain $\Gamma_{1}^{{ }^{p-n}}\left(Q^{2}\right)$ at 2.0 and $4.6 \mathrm{GeV}^{2}$. Inserting their results into equation 15 gives

$$
\begin{aligned}
& I_{1}^{p-n}\left(Q^{2}=2.0 \mathrm{GeV}^{2}\right)=0.143 \pm 0.013 \pm 0.020 \\
& I_{1}^{p-n}\left(Q^{2}=4.6 \mathrm{GeV}^{2}\right)=0.082 \pm 0.020 \pm 0.008
\end{aligned}
$$

At very large $\mathrm{Q}^{2}, I_{1}^{p-n}\left(Q^{2}\right)$ scales with $1 / Q^{2}\left(\Gamma_{1}^{p-n}\left(Q^{2}\right)\right.$ is a constant). Were this same $\mathrm{Q}^{2}$ dependence to connect these finite $\mathrm{Q}^{2}$ measurements with the $\mathrm{Q}^{2}=0$ (DHG) value, as had been suggested by Anselmino et al.(AIL89), it would indicate that the higher twist corrections to the structure function sum rules for the proton and neutron exactly cancel. A comparison of equations 16 and 17 makes it clear that such a simple extrapolation from finite $\mathrm{Q}^{2}$ is not consistent with the DHG prediction at $\mathrm{Q}^{2}=0$. Furthermore, this disagreement persists even when twist-4 corrections are included(EK93). This is illustrated 


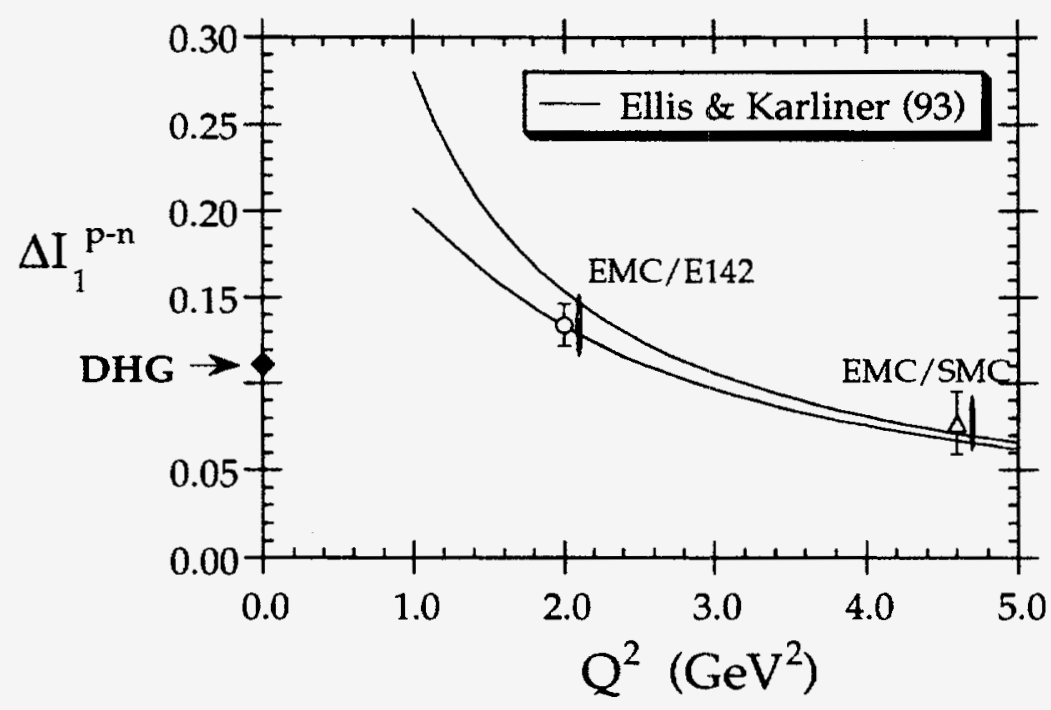

Figure 1. $I_{1}^{p-n}\left(Q^{2}\right)$ as a function of $Q^{2}$. The combined EMC/E142 (circle) and EMC/SMC (triangle) data shown with statistical errors(EK93). The associated systematic errors are indicated by the vertical bars to the right of each point. The upper and lower limits of the error band of a calculation including twist-4 corrections ${ }^{(\mathrm{EK} 93)}$ is shown as the solid lines.

in figure 1. It appears that if equation 16 is correct, there is a significant difference between the proton and neutron spin structure in the small $Q^{2}$ region.

It is interesting to note that Bernard, Kaiser and Meißner(BKM93) also find that in relativistic $\chi \mathrm{PT}$ the slopes of $I_{1}^{p}\left(Q^{2}\right)$ and $I_{1}^{n}\left(Q^{2}\right)$ at $\mathrm{Q}^{2}=0$ are quite different, again suggesting a difference between the proton and neutron spin structures.

\section{The Role of the 4 (1232) Resonance}

The baryon resonances, particularly the $\Delta(1232)$ resonance, can potentially have a large impact on the difference of the spin-dependent cross sections that enter the spinpolarizability and DHG integrals. The role of the $\Delta(1232)$ can be readily understood from the almost pure magnetic dipole character of the excitation. The M1 operator drives spinflip transitions and, to the extent that the nucleon spin is carried by s-wave constituent quarks, the $\Delta$ can contribute only when the photon and nucleon spins are parallel. Thus, for small $\mathrm{Q}^{2}$ (in particular at $\mathrm{Q}^{2}=0$ ), we expect $\sigma_{3 / 2} \gg \sigma_{1 / 2}$. This would make both $\gamma$ and the inelastic contribution to $I_{1}^{p}\left(Q^{2}\right)$ large and negative. On the other hand, the requirement of chiral invariance causes the amplitude for $\Delta$-electroproduction to decrease rapidly ${ }^{(\mathrm{Bu} 93)}$ and become negligible at large $Q^{2}$, which implies that $I_{1}^{p}\left(Q^{2}\right)$ becomes positive. Thus, one expects the $\Delta$ to contribute to the change in the sign of $\mathrm{I}_{1}\left(\mathrm{Q}^{2}\right)$. Although the work by Ji(ji93) 
shows increasingly large (and negative) contributions from virtual Compton scattering as $\mathrm{Q}^{2}$ decreases, the Compton terms for the proton are in fact non-analytic and vanish at exactly $Q^{2}=0$, while for the neutron there is an accidental numerical cancellation in the form factors of eq. (13a) that zeros the elastic contribution as $Q^{2} \rightarrow 0$. Thus, for both the proton and the neutron, at the real photon point the $\Delta$ drives $I_{1}$ towards large negative values, as expected in eq. (13b).

Burkert and $\mathrm{Li}^{(\mathrm{Bu} 93)}$, computing the inelastic contribution to $I_{1}$, have used existing $\pi$ and $\eta$ electroproduction data from the proton to parameterize the amplitudes for the excitation of resonances up to $2 \mathrm{GeV}$. Assuming these resonances dominate the cross section, they observe a change in the slope and the sign of both $I_{1}^{p}$ and $I_{1}^{p-n}$ at small values of $\mathrm{Q}^{2}$, most of which appears to be due to the behavior of the amplitude for $\Delta$ production. As shown in figure 2, these calculations do not obviously connect to the DHG point. The proton-neutron difference depends on details that clearly cannot be extracted from existing data.

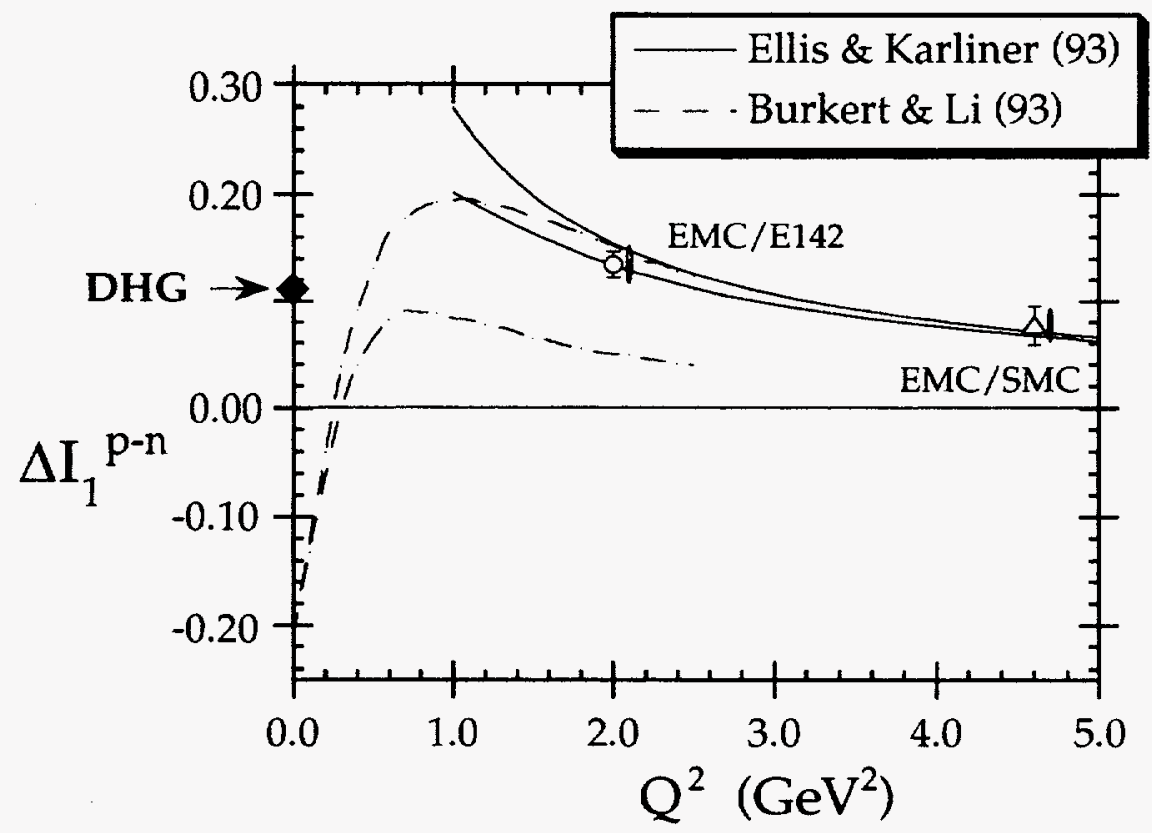

Figure 2. $I_{1}^{p-n}\left(Q^{2}\right)$ as estimated by Burkert ${ }^{(B u 93)}$. The dashed curves result from a parameterization of single-meson production from $\mathrm{N}^{*}$ resonances up to $2.0 \mathrm{GeV}$ The two different curves follow from different model assumptions used to conpensate for the sparce neutron data. (See legend to figure 1.)

Because the spin-dependent polarizability and DHG integrals both depend on the same helicity-dependent differences, the large contributions of the $\Delta$ to the DHG sum rule will also be apparent in evaluating $\gamma$ from equation 4 . In fact, $\gamma$ will be even more sensitive to the effect of the $\Delta$ due to the suppression of the higher-lying resonances by the $1 / \omega^{3}$ weighting. 


\section{Estimates from Photoproduction Multipoles}

In absence of direct observation of the helicity-dependent cross sections, it is possible to make an estimate of the spin-dependent polarizability and the DHG sum rule integrals using existing multipole analyses, constructed from measurements (mostly unpolarized) of the different charge channels in meson production. To do this, an isospin decomposition of the integrals is made, producing three components: the isovector (vv), isoscalar (ss) and mixed (vs) terms. For the spin-dependent polarizability this decomposition gives:

$$
\begin{aligned}
& \gamma_{v v}=\int_{\omega_{0}}^{\infty}\left[\sigma_{y / 2}^{v V}-\sigma_{3 / 2}^{v V}\right] \frac{d \omega^{\prime}}{\omega^{\prime 3}} \\
& \gamma_{v S}=\int_{\omega_{0}}^{\infty}\left[\sigma_{y / 2}^{v s}-\sigma_{3 / 2}^{v s}\right] \frac{d \omega^{\prime}}{\omega^{\prime 3}} \\
& \gamma_{s s}=\int_{\omega_{0}}^{\infty}\left[\sigma_{y_{2}}^{s S}-\sigma_{3 / 2}^{s s}\right] \frac{d \omega^{\prime}}{\omega^{\prime 3}}
\end{aligned}
$$

so that $\gamma_{p, n}=\gamma_{V V}+\gamma_{s S} \pm \gamma_{V S}$. Assuming that one- $\pi$ production is the dominant process, the $3 / 2$ and $1 / 2$ helicity cross sections for the proton and neutron have been estimated(SWK94), using the $\mathrm{N}(\gamma, \pi)$ multipoles (VPI-FA93) of Workman and Arndt(WA93). The importance of the $\Delta$, as noted in the previous section, makes the VV contribution the largest. The known $\pi \pi N / \pi N$ branching ratios of $N^{*}$ resonances have been used to estimate $2 \pi$ photoproduction. This $2 \pi$ contribition is a small correction here since the $\Delta$ has no $\pi \pi \mathrm{N}$ branch and the effect of the higher-lying $N^{*}$ resonances is drastically reduced by the $1 / \omega^{3}$ weighting of the integrals in Eq. (18). The results are given in Table 1, together with the predictions of Bernard et al. (BKKM92) from Chiral Perturbation Theory $(\chi \mathrm{PT})$.

Table I. Estimates of the nucleon spin-dependent polarizability(SWK94), using the VPI-

\begin{tabular}{|c|c|c|c|}
\hline & \multirow{2}{*}{$\begin{array}{l}\text { Multipole } \\
\text { estimate } \\
\text { FA93 }\end{array}$} & \multicolumn{2}{|c|}{$\chi \mathbf{P T}$} \\
\hline & & $\begin{array}{c}\text { relativistic } \\
1-\text {-loop }\end{array}$ & 1-loop $+\Delta$ \\
\hline$\gamma_{p}$ & -134 & +216 & -150 \\
\hline$\gamma_{n}$ & -38 & +320 & -46 \\
\hline$\gamma_{V S}$ & -48 & -52 & -52 \\
\hline
\end{tabular}
FA93 multipole analysis(WA93), compared with the $\chi$ PT prediction of Bernard et al. (BKKM92). Tabulated values are in units of $10^{-6} \mathrm{fm}^{4}$. 


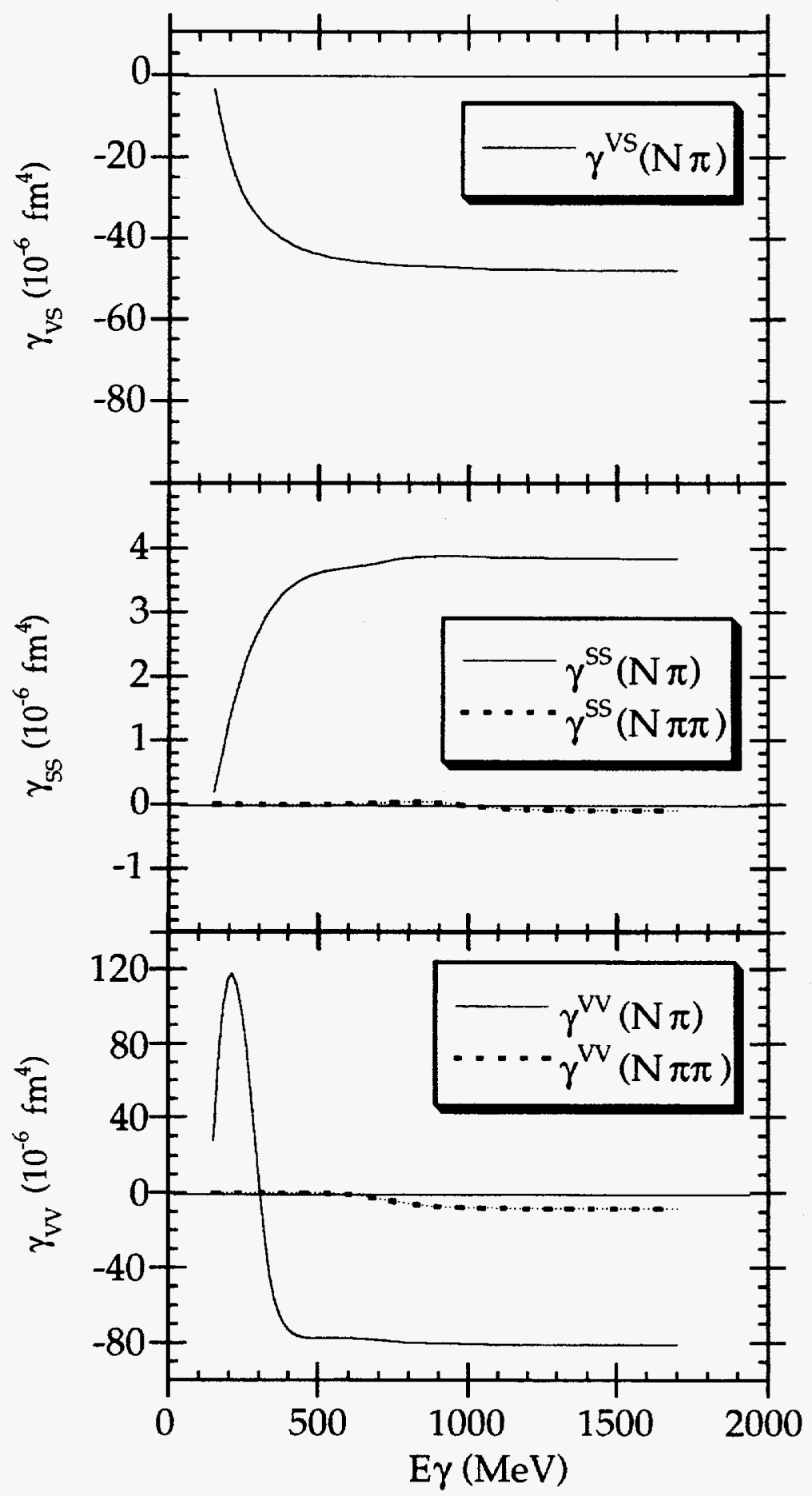

Figure 3. The Isospin decomposition of the spin-dependent polarizability, $\gamma$, into vector (VV), scalar (SS), and mixed (VS) components (SWK94), using the VPI-FA93 amplitudes (WA93), plotted as a function of the upper limit of integration. The solid and dotted curves give the contributions of the $N \pi$ and $N \pi \pi$ channels, respectively. 
The isospin decomposition of the one- and two-pion production contributions to $\gamma$ as a function of the upper limit of the integral are show in figure 3. The $N \pi \pi$ contribution is small for all three components and by $500 \mathrm{MeV}$ the integrals have essentially converged. The importance of the $\Delta$ is clearly seen in $\gamma_{V V}$, and the large $\gamma_{v s}$ contribution reflects the large proton-neutron difference. Combining the values at $1.7 \mathrm{GeV}$ gives the results summarized in Table 1.

The relativistic 1-loop $\chi \mathrm{PT}$ predictions listed in Table 1 , as well as the nonrelativistic heavy-baryon calculations of Bernard et al.(BKKM92), differ significantly from those of the FA93 multipoles. However, Bernard et al. have estimated the effect of the $\Delta$ and found it to be significant and negative $\left(\gamma_{p, n}^{4}=-366 \times 10^{-6} \mathrm{fm}^{4}\right)$, thereby bringing the $\chi \mathrm{PT}$ proton and neutron values much closer to those of the multipole analysis. Encouraging as this is, verification must await a complete calculation including all $\Delta$ effects to the same order. Nonetheless, since the $\Delta$ contribution in $\chi \mathrm{PT}$ is the same for the proton and neutron, the Vector-Scalar component, $\gamma_{v s}=1 / 2\left(\gamma_{p}-\gamma_{n}\right)$, is unaffected by the $\Delta$, and here relativistic 1-loop $\chi \mathrm{PT}$ is in impressive agreement with FA93.

The DHG sum rule (eq. 8) may also be estimated using the FA93 multipole analysis. Here, isospin decomposition gives,

$$
\begin{aligned}
& D H G_{V V}=\int_{\omega_{0}}^{\infty}\left[\sigma_{y_{2}}^{V V}-\sigma_{3 / 2}^{V V}\right] \frac{d \omega^{\prime}}{\omega^{\prime}}=-\left(\frac{1}{2} \kappa_{V}\right)^{2} \frac{2 \pi^{2} \alpha}{M^{2}} \\
& D H G_{V S}=\int_{\omega_{0}}^{\infty}\left[\sigma_{y_{2}}^{V S}-\sigma_{3 / 2}^{V S}\right] \frac{d \omega^{\prime}}{\omega^{\prime}}=-\frac{1}{2} \kappa_{V} \kappa_{S} \frac{2 \pi^{2} \alpha}{M^{2}} \\
& D H G_{S S}=\int_{\omega_{0}}^{\infty}\left[\sigma_{y / 2}^{S S}-\sigma_{3 / 2}^{S S}\right] \frac{d \omega^{\prime}}{\omega^{\prime}}=-\left(\frac{1}{2} \kappa_{S}\right)^{2} \frac{2 \pi^{2} \alpha}{M^{2}}
\end{aligned}
$$

where $\kappa_{V}$ and $\kappa_{s}$ are related to the anomalous magnetic moments of the proton and neutron by

$$
\begin{aligned}
& \kappa_{P}=\frac{1}{2}\left(\kappa_{S}+\kappa_{V}\right) \\
& \kappa_{N}=\frac{1}{2}\left(\kappa_{S}-\kappa_{V}\right)
\end{aligned}
$$

The first estimates of these three terms were made by Karliner(Ka73), assuming that $\pi$-production dominated the total cross section. This was performed using a multipole analysis of mostly unpolarized data. There have been several measurements of polarization observables in recent years (although no direct determinations of the helicitydependent terms that enter eq. 19), and these have been included in recent multipole analyses. The calculation of the DHG integrals has been repeated using the VPI-SP92 multipoles(WA92), and more recently using the VPI-FA93 multipole solution(SWK94). Results from the latter are shown in figure 4 where the isospin decomposition of cross section differences (dashed curves) is plotted as a function of photon energy, as well as the sum rule integrals (solid curves), plotted against the upper energy limit of integration. 


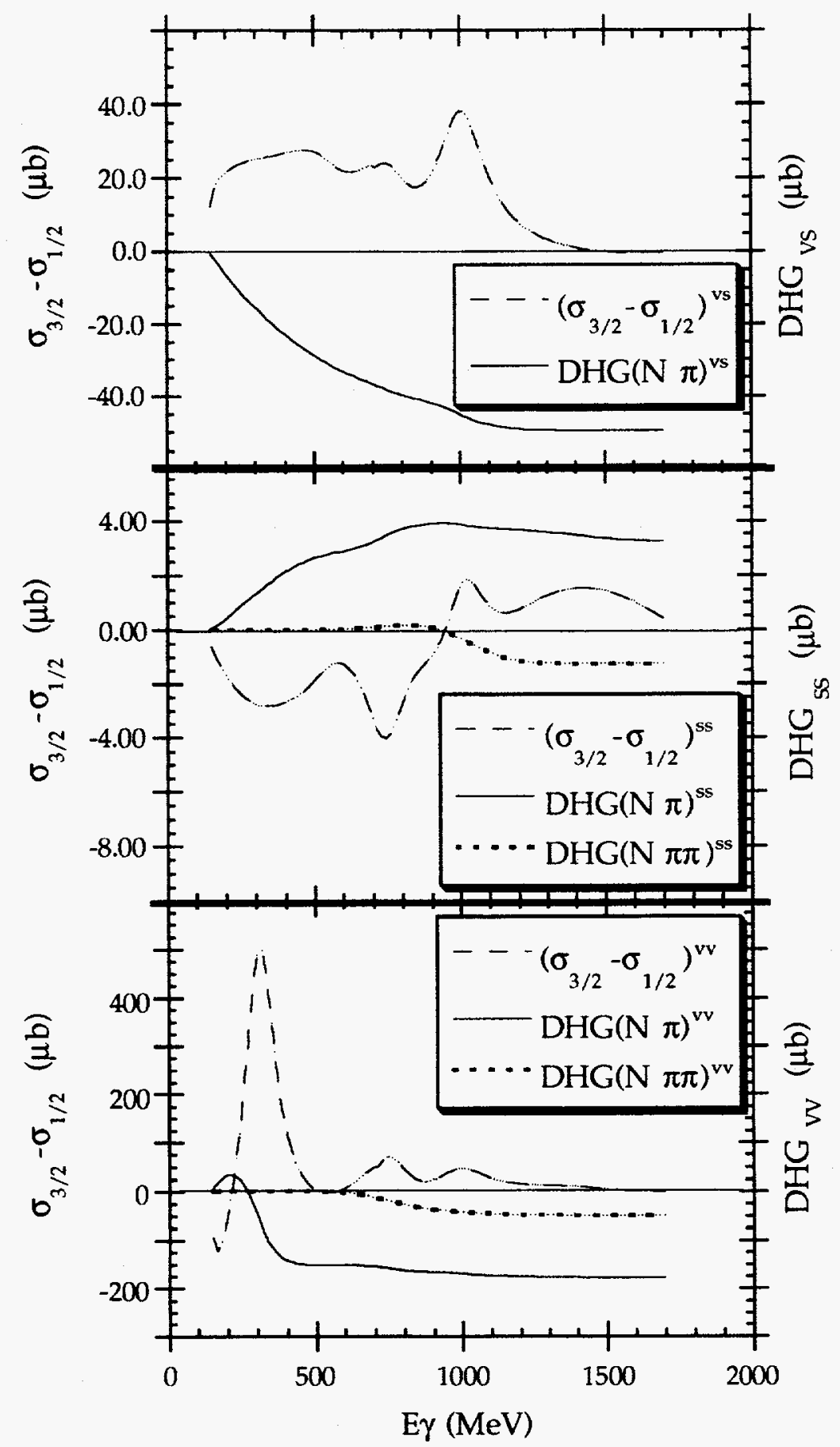

Figure 4. The Isospin decomposition of the DHG integral into VV, SS, and VS components (SWK94), using the VPI-FA93 multipoles(WA93). The dashed curves give the difference of the spin-dependent cross sections, $\Delta \sigma_{i j}=\left(\sigma_{3 / 2}-\sigma_{12}\right)$ ij, for single-pion production. The solid and dotted curves give the contributions to the DHG integral from $N \pi$ and $N \pi \pi$ channels, respectively. The latter have been calculated by scaling the $\mathrm{N} \pi$ results with the known $N \pi \pi / N \pi$ branching ratios for $N^{*}$ resonances. Units are in $10^{-4} \mathrm{fm}^{2}(\mu \mathrm{b})$. 
Table 2. The Isospin decomposition of the DHG integral(SWK94), as estimated from photo-production multipoles, in units of $10^{-4} \mathrm{fm}^{2}(\mu \mathrm{b})$.

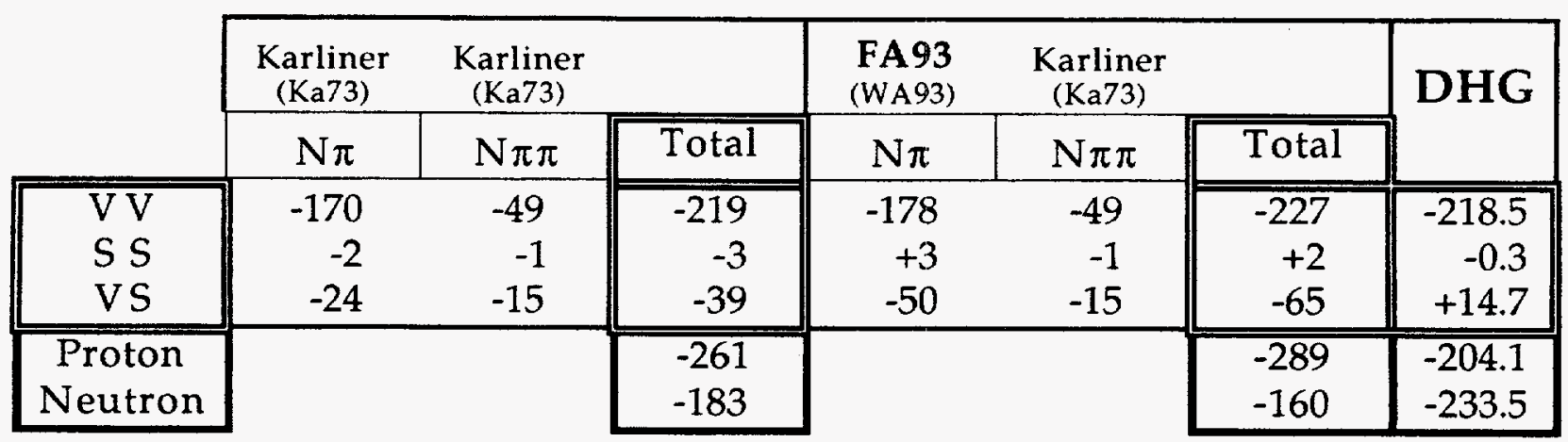

The predictions of the FA93 multipoles are summarized in table 2 for the three isospin components of the DHG integral(SWK94), together with the original Karliner analysis(Ka73). The right-most column gives the results expected from the magnetic moments in the right-hand side of eqn. (19). The large isovector (VV) component obtained in these analyses from the $N \pi$ multipoles appears to be quite stable. Combining this with the estimate for the $N \pi \pi$ contribution gives quite reasonable agreement with the sum rule value. Similarly, the total isoscalar (SS) contribution $(N \pi+N \pi \pi)$ is consistently small, as is the corresponding DHG value.

The total multipole estimates from the recent FA93 solution, for both the proton and the neutron, are $40 \%$ different from the full DHG sum rule predictions, and this discrepancy is almost entirely due to the Vector-Scalar (VS) contribution which differs in magnitude from the magnetic-moment value by a factor of 4 , and is of the opposite sign. (Here the VS component from FA93 is somewhat larger than that of the SP92 solution reported in ref. WA92.) As can be seen in figure 4, almost $2 / 3$ of the DHG value is saturated in integrating up to $500 \mathrm{MeV}$, largely because of the $1 / \omega$ energy weighting. This is the energy region containing the greatest concentration of published measurements and, thus, it is precisely the region where multipole analyses would be expected to be the most reliable.

The earlier analysis of Karliner also predicted a large negative $D H G_{V S}$ contribution, $-39 \mu \mathrm{b}$ (table 2). Here, the disagreement with the $+15 \mu \mathrm{b}$ value from the sum rule, although still appreciable and of opposite sign, is less than that fof FA3. The values for $\Delta \sigma^{v s}=\left[\sigma_{3 / 2}-\sigma_{1 / 2}\right]^{v s}$ predicted by these multipoles are shown in figure 5 . The main differences between the Karliner(Ka73) and FA93 results lie in the region between 400 and $600 \mathrm{MeV}$, and occur mostly in the contributions from charged- $\pi$ production. In the absence of direct measurements, an unambiguous prediction of the $\Delta \sigma=\left(\sigma_{3 / 2}-\sigma_{1 / 2}\right)$ difference requires accurate knowledge, in both isospin channels, of 7 quantities: the unpolarized cross section, the 3 single-polarization observables, and a minimum of 3 out of 12 possible double-polarization observables(BDS75). There have been many measurements of spin-observables in recent years, although a complete set is still lacking. 


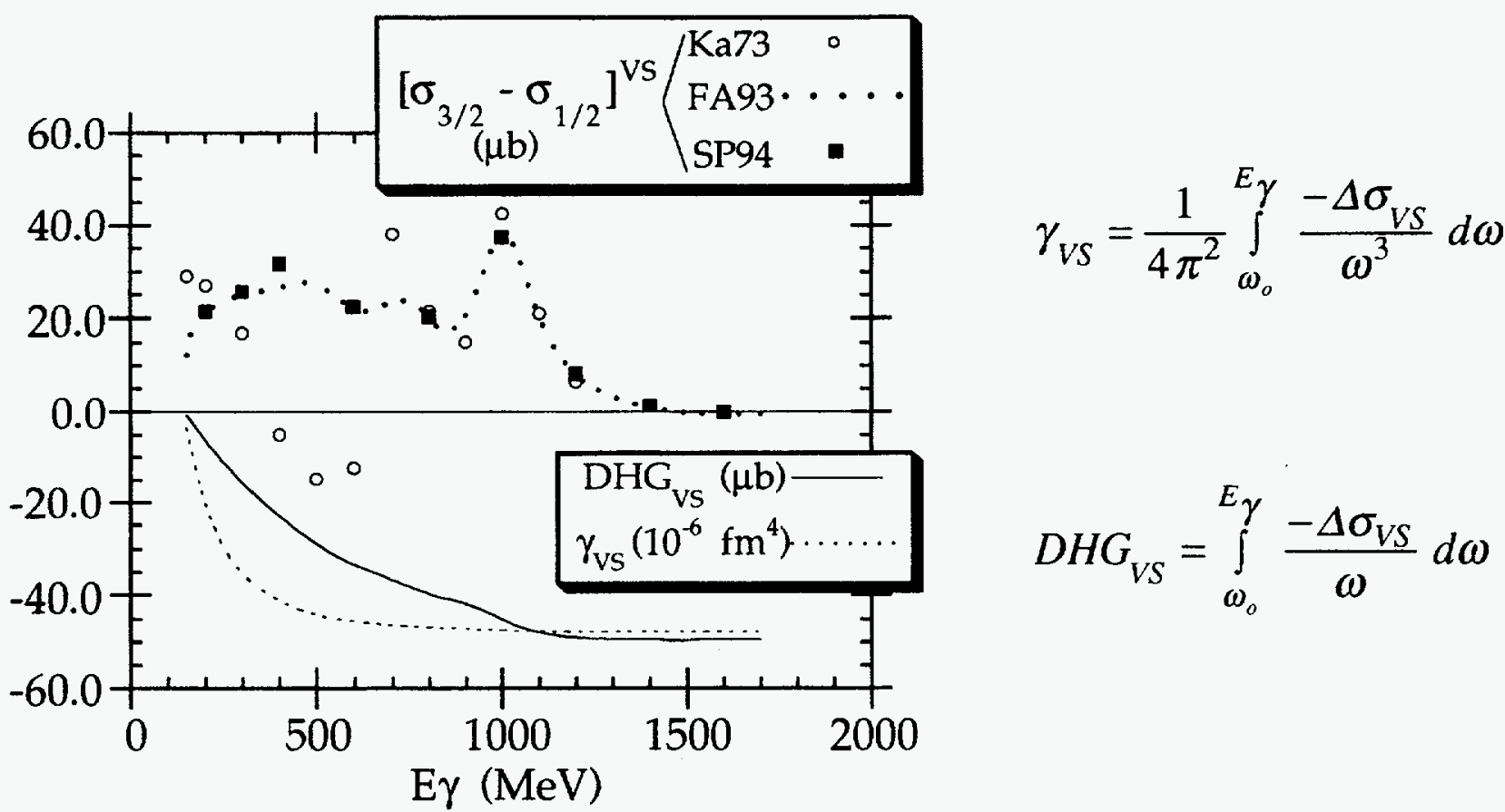

Figure 5. The predictions for $\Delta \sigma=\left(\sigma_{3 / 2}-\sigma_{1 / 2}\right)$, for the proton-neutron difference $\Delta \sigma^{V S}=1 / 2\left(\Delta \sigma_{p}-\Delta \sigma_{n}\right)$, in $\mu \mathrm{b}$, as computed by Karliner(Ka73), and by (SWK94) using the FA93 and SP94 multipoles from VPI(WA93). The VS contributions to the $\gamma$ and DHG integrals, computed with FA93, are shown in the lower curves as a function of the upper energy-limit of integration.

However, there was almost no information on polarization degrees of freedom at the time of the 1973 Karliner analysis, and this is the main limitation of that work. Figure 5 also shows the predictions of a new VPI solution, SP94. The chief difference between FA93 and SP94 is the recent inclusion in the VPI data base of large sets of high precision singlepolarization data from the LEGS and Bonn facilities. Nonetheless, the results for $\Delta \sigma^{V S}=\left[\sigma_{3 / 2}-\sigma_{1 / 2}\right]^{V S}$ are almost unchanged. Further refinements will require doublepolarization data.

\section{Consequences of the Incompatibility of Predictions for the Proton-Neutron Difference}

The FA93 predictions for $\gamma_{v s}$ and $D H G_{v s}$ are plotted together in figure 5 as a function of the upper integration bound. Their apparent convergence leaves little room for reconciling the predictions for these quantities. The multipole calculations for the $D H G_{V S}$ integral (table 2) consistently predict the opposite sign (negative) and a significantly larger magnitude than the sum rule of eqn. (19). But, this is in sharp contrast to the FA93 prediction for $\gamma_{v S}$ (table 1) which is within $8 \%$ of the relativistic 1-loop $\chi$ PT value. In 
principal, the different energy weightings of the $\gamma_{v s}$ and $D H G_{v s}$ integrals admit the possibility that contributions above the $1.7 \mathrm{GeV}$ limit of the VPI data base could bring the $D H G_{V S}$ value up to that expected by the sum rule, without appreciably affecting $\gamma_{v s .}$ However, $1.7 \mathrm{GeV}$ is already so large that such $\Delta \sigma^{V S}=1 / 2\left(\Delta \sigma_{p}-\Delta \sigma_{n}\right)$ differences would have to be huge in order to overcome the $1 / \omega$ DHG energy weighting. For example, $\Delta \sigma^{V S}=-200 \mu b$ between 2 and $3 \mathrm{GeV}$, which would require a prominent but as yet unidentified resonance; or a constant level of $\Delta \sigma^{V S}=-20 \mu b$ extending out to $100 \mathrm{GeV}$, which would be much larger than the contributions of the resonance region of figure 5 in which the isospin structure of the $\Delta$ and $\mathrm{N}^{*}$ states can be expected to enhance the protonneutron difference. Furthermore, in either case, the $1 / 2\left(\Delta \sigma_{p}+\Delta \sigma_{n}\right)$ sum would have to remain unaffected so as not to destroy the agreement with the isovector and isoscalar components in table 2. Apart from such scenarios, which seem highly unlikely, there are only two other possibilities. Either (a) both the 2-loop corrections to the Spin-Polarizability are large and the existing multipoles are wrong, or (b) modifications to the Drell-HearnGerasimov sum rule are needed to fully describe the isospin structure of the nucleon.

It is possible that the 2-loop corrections to the $\chi P T$ calculation of $\gamma$ are large. Although this is not usually the case for $\chi P T$ expansions, it would not be without precedence. Since the $\mathrm{N} \pi \pi$ contributions do not appear to be large, a significant 2-loop component to $\gamma$ would then imply that the $\mathrm{N} \pi$ multipoles require modifications. Although such modifications could alter the predictions for several other observables, the effects might also be quite subtle. To recover the DHG values in table 2, $D H G_{p}$ would have to increase by $\sim 80 \mu \mathrm{b}$ while $D H G_{n}$ decreased by the same amount. If this were achieved by changing $\sigma_{1 / 2}$ and $\sigma_{3 / 2}$ by amounts of equal magnitude but of opposite sign, for both the proton and the neutron, then the unpolarized cross sections, and related sum rules such as eqn. (3) for the nucleon polarizabilities, would remain in agreement with experiment. Nonetheless, even in such complicated scenarios other double-polarization observables would certainly be affected, notably the beam-recoil asymmetry, $C_{z}$, and the target-recoil asymmetry, $L_{z}$, which, like $\Delta \sigma$ and the unpolarized cross section, are constructed from different combinations of the squares of the helicity amplitudes (BDS75). Unfortunately, there are as yet no measurements of these quantities.

Alternatively, if the multipoles are basically correct, then the DHG sum rule requires a modification of the form

$$
D H G_{V S}=\frac{1}{2} \int_{\omega_{0}}^{\infty} \frac{\left[\sigma_{1 / 2}-\sigma_{3 / 2}\right]_{p}-\left[\sigma_{1 / 2}-\sigma_{3 / 2}\right]_{n}}{\omega} d \omega=-\frac{1}{2}\left(\kappa_{p}^{2}-\kappa_{n}^{2}\right) \frac{2 \pi^{2} \alpha}{m^{2}}-C
$$

The simplest choice for the correction factor needed to bring the VS sum rule down to the FA93 values of table 2 would be $C=2 \pi^{2}\left[g_{p}(\infty)-g_{n}(\infty)\right]$, with $g_{p}(\infty) \approx-g_{n}(\infty) \approx 2 \mu b$ so as to preserve the existing agreement in the VV and SS components. In other words, contrary to the original DHG assumption that $g(\infty)=0$ in eqn. (8a), $g_{p}$ and $g_{n}$ would tend to nearly equal but opposite constants at high energy. The physical origin of such constants would be quite interesting. Abarbanel and Goldberger, and Fox and Freedman, (FF69) have shown 
that such a situation can result from a $J=1$ fixed pole in the angular momentum plane, but there may be other explanations. In addition, the $D H G_{v S}$ integral is just the $Q^{2}=0$ limit of the Bjorken sum rule integral ${ }^{(B j 66)}$, and $C=0$ has been assumed in modeling its $Q^{2}$ evolution(AIL89,BL93,Bu93,ST93,Ji93). The $Q^{2}$ dependence of a possible non-zero $g(\infty)$ remains to be considered.

On the other hand, changes in the DHG sum rule due to QCD-current algebra effects, arising from possible corrections to the commutator of the charge densities generated by the quark fields, have also been proposed(KS66,CLW94). Estimating such a modification involves a number of assumptions, which are minimized in the protonneutron difference. In a recent work, Chang, Liang and Workman have proposed a modified $D H G_{v S}$ sum rule with $C=\alpha g_{A} / 6 F_{\pi}^{2}$ in eqn. 21 . Once again, $g_{A}=1.25$ is the axialvector coupling constant and $F_{n}=93 \mathrm{MeV}$ is the pion $\beta$-decay constant. This term would bring the expected $D H G_{v S}$ value reasonably close $(-54 \mu \mathrm{b})$ to the multipole predictions of table 2 . However, as pointed out by Kawarabayashi and Suzuki(KS66), such current-algebra modifications could potentially change the LGG low-energy theorem and it remains to be checked that eqn. (2c) is preserved in such schemes.

In summary, there is a significant incompatibility between the $\chi P T$ calculation for the nucleon spin polarizability, the evaluation of the conventional DHG sum rule, and the predictions for these quantities using recent multipole analyses. If the 2-loop corrections to $\gamma$ are indeed large, it will be quite important to provide an experimental constraint since such calculations are quite demanding. Alternatively, if the corrections to the DHG sum rule are large, a determination of the $D H G_{v S}$ integral could provide a unique constraint on the high-energy spin-dependent Compton amplitude, and on possible components of the quark currents that have previously remained elusive. Ultimately, this situation can only be resolved with direct measurements of the $\sigma_{1 / 2}$ and $\sigma_{3 / 2}$ cross sections on both the proton and the neutron. Since the key physics issues with the least model dependence are in the proton-neutron difference, each of which involve cross section differences themselves, considerable care must be taken to minimize systematic uncertainties. The SPHICE target described in the next section is uniquely suited to such measurements. 
SPHICE:

\section{A Strongly Polarized IHydrogen and Deuterium IICE Target}

The combination of the LEGS polarized photon beams and a new polarized target, SPHICE, providing both polarized protons and polarized deuterons (and hence, neutrons), is uniquely suited to measurements of the helicity-dependent cross sections that determine the $\gamma$ and DHG integrals of eqs. 4 and 8 .

The ideal polarized hydrogen or deuterium targets for nuclear physics experiments would consist of pure hydrogen or deuterium. However, because of the small nuclear moments, this requires the use of very low temperatures $(10 \mathrm{mK})$ and high magnetic fields $(>10$ Tesla). These conditions, especially the large magnetic fields, make pure targets impractical. Moreover, the limitations imposed by wave function symmetry in the homonuclear $\mathrm{H}_{2}$ molecule mean that the proton spins (in the ground state) are antialigned at $\mathrm{T}=0^{\circ} \mathrm{K}$, and pure hydrogen cannot be polarized at all.

Two solutions have been developed to overcome these limitations. Frozen spin targets have been produced in a metastable polarized state that will persist for hours or days after the polarizing conditions have been removed. In this way, the experimental and polarizing conditions may be different. Alternatively, dynamical nuclear polarization (DNP) has been used to maintain the target polarization by a continuously applied Rf-field. This latter method requires more modest fields and temperatures and is compatible with experimental conditions. However, both methods produce targets that contain only $1 / 3$ to $1 / 4$ of the species of interest, and partial polarization of the remaining species complicate the situation even further. (Existing targets are usually made with $\mathrm{NH}_{3}$ and $\mathrm{ND}_{3}$.)

SPHICE, a Strongly Polarized Hydrogen and Deuterium ICE Target, represents a new technology utilizing molecular HD in the solid phase. Once polarized, the coupling between the nucleon spin and the atomic lattice, which is primarily through molecular rotations, causes the spin alignment to relax back to the unpolarized state. This represents the chief limitation on conventional 'frozen-spin' targets. However, the heteronuclear HD molecule, consisting of non-identical particles, does not have any restrictions on the orbital and spin angular momenta and hence can be in the molecular-rotation state $\mathrm{J}=0$, independent of the $\mathrm{H}$ or $\mathrm{D}$ spin orientations at low temperature. Thus, the spin-lattice coupling, and the corresponding relaxation rates, are exceedingly small and imply a sample whose spins would remain 'frozen' for many days even at $4^{\circ} \mathrm{K}^{(\mathrm{Ho} 89)}$.

\section{Hydrogen polarization by High-Field Relaxation with Ortho- $\mathrm{H}_{2}$ Doping}

The symmetry restrictions imposed on the total wave functions of $\mathrm{H}_{2}$ ( $t$ wo fermions) and $\mathrm{D}_{2}$ (two bosons) limit the possible nuclear spin orientations at low temperature. For hydrogen at room temperature, the most abundant species, the so-called ortho state, is nuclear spin $\mathrm{I}=1$ and molecular orbital angular momentum $\mathrm{L}=$ odd. Since the nuclear spins are aligned, $\mathrm{o}-\mathrm{H}_{2}$ can be polarized. However, the equilibrium condition at low temperature is the para-hydrogen state with $\mathrm{I}=0$ and $\mathrm{L}=0$. Here the nuclear magnetic moments are antiparallel and the sample cannot be polarized. In the case of deuterium, it is the $p-D_{2}$ combination $(I=1 ; L=o d d)$ that disappears at low temperature, leaving the $o-D_{2}$ 
$(I=0,2 ; L=0)$. Although $5 / 6$ of the nuclei in this state have their spins parallel $(I=2)$, the small magnetic moment of the deuteron makes polarization of pure deuterium very difficult.

In contrast, the orbital and spin angular momenta of the heteronuclear molecule HD is not limited by symmetry requirements and hence can be in the molecular-rotation state $L=0$ independent of the $\mathrm{H}$ or $\mathrm{D}$ spin orientations at low temperature. Since the spinlattice coupling is primarily by way of the molecular rotations, the relaxation rates are exceedingly small, corresponding to relaxation times of days even at $4^{\circ} \mathrm{K}^{(\mathrm{Ho} 89)}$ Although this long relaxation time is essential for usable targets, it makes the polarization phase equally long. The solution to this problem was suggested by Honig(Ho67) in 1967. The relaxation (polarization) time can be reduced by introducing small $\left(\sim 10^{-4}\right)$ concentrations of $\mathrm{o}-\mathrm{H}_{2}$ and $\mathrm{p}-\mathrm{D}_{2}$.

The presence of $\mathrm{o}-\mathrm{H}_{2}$ and $\mathrm{p}-\mathrm{D}_{2}$ are important for the polarization process since the molecular orbital angular momentum couples with both the lattice and the nuclear spin. The transistions from $\mathrm{o}-\mathrm{H}_{2}$ to $\mathrm{p}-\mathrm{H}_{2}$, and from $\mathrm{p}-\mathrm{D}_{2}$ to $\mathrm{o}-\mathrm{D}_{2}$, occur with time constants of 6.25 and 18.25 days, respectively. These rates are independent of temperature and applied magnetic field. Thus, for $\mathrm{H}_{2}$ and $\mathrm{D}_{2}$ the spin-lattice coupling that makes polarization (and depolarization) possible is time dependent.

Although the $\mathrm{o}-\mathrm{H}_{2}$ and $\mathrm{p}-\mathrm{D}_{2}$ decay rates are independent of temperature and applied magnetic field, the polarization relaxation times for the hydrogen and deuterium in HD are a function of the $\mathrm{o}-\mathrm{H}_{2}$.and $\mathrm{p}-\mathrm{D}_{2}$.concentrations as well as the temperature and magnetic field. The hydrogen and deuterium relaxation times are long for small $o-\mathrm{H}_{2}$ and $\mathrm{p}-\mathrm{D}_{2}$ concentrations and large magnetic fields $(\mathrm{Ho} 89)$. Thus, by doping the pure $\mathrm{HD}$ with $\mathrm{o}-\mathrm{H}_{2}$ and $p-D_{2}$, the relaxation times of the $H$ and $D$ in HD are reduced so that the sample may be polarized. To produce a practical target, the relaxation times must be increased after the target is polarized so that it is in a metastable state and can be removed from the high field/low temperature conditions for use as a target.

The choice of $o-\mathrm{H}_{2}$ and $p-\mathrm{D}_{2}$ as the magnetically active impurities provides a natural mechanism for achieving this since they decay to the magnetically inert $\mathrm{p}-\mathrm{H}_{2}$ and $\mathrm{o}-\mathrm{D}_{2}$. This provides a so-called "relaxation switch": by simply holding the target at the low temperature and high field conditions, the relaxation times will increase as the impurity concentration declines.

Thus, to begin the polarizing cycle a suitably doped HD sample is held at high magnetic field and low temperature. The proton and deuteron spins relax, aligning with the magnetic field and transferring the transition energy to the lattice. On a time scale set by the initial doping concentrations, the equilibrium polarization is achieved. The equilibrium (vector) polarization for nuclei of spin I is given as a function of the magnetic field (B) and the temperature $(\mathrm{T})$ by the Brillouin function

$$
P_{V}(B / T)=\frac{2 I+1}{2 I} \operatorname{coth}\left\{\frac{(2 I+1) g \mu_{N} B}{2 k T}\right\}-\frac{1}{2 I} \operatorname{coth}\left\{\frac{g \mu_{N} B}{2 k T}\right\} \quad,
$$


where $\mathrm{g}$ is the nuclear $\mathrm{g}$-factor, $\mathrm{k}$ is the Boltzman constant and $\mu_{N}$ is the nuclear magneton. Since the populations of the magnetic sub-states are characterized by a temperature which determines the Boltzman factor, $e^{-E / k T}$, the vector and tensor polarization (or alignment) of the deuteron are not independent ${ }^{(\mathrm{Ki})}$, but are related by

$$
P_{\tau}=2-\sqrt{4-3 P_{v}^{2}}
$$

Since the polarization depends only on the ratio of $B / T$, the production of high polarizations by relaxation requires the use of a dilution refrigerator and a superconducting magnet. The equilibrium polarizations that can be achieved in this way with the SPHICE target, cooled to $15-20 \mathrm{mK}$ in a magnetic field of $17 \mathrm{Tesla}$, are $80 \%$ for hydrogen and $20 \%$ (vector) for deuterium. The sample must be held at these temperature and field conditions for several o- $\mathrm{H}_{2} / \mathrm{p}-\mathrm{D}_{2}$ time constants until these magnetic impurities have decayed to the point that the target spin is effectively "frozen".

Although proton polarizations obtained in this way are quite high, the deuteron polarization is only $20 \%$. Furthermore, while the 6.25 day decay constant for the $\mathrm{o}-\mathrm{H}_{2}$ impurity requires high-field/low-temperature holding times of a few weeks, the corresponding 18.2 day decay constant for the magnetically active $p-D_{2}$ would require holding periods of months. Holding periods of a few weeks are manageable, but several months are rather impractical.

\section{Deuteron polarization by Adiabatic Fast Passage}

In the standard cycle that will be used to prepare SPHICE targets, $\mathrm{o}-\mathrm{H}_{2}$ doping will be used to polarize the hydrogen at high field/low temperature, but no $p-D_{2}$ will be introduced. Rather, the deuterium will be polarized by successively transfering the hydrogen polarization to the deuterons with a method commonly known as 'Adiabatic Fast Passage'(HM76). This technique takes advantage of the dipolar coupling of $H$ and $D$ nuclei in different HD molecules. The energy level diagram for the coupling of a single $\mathrm{H}$ and a single $\mathrm{D}$ on neighboring HD molecules is shown in figure 6.

Although the states, labeled a-f, can be approximately characterized by the high field quantum numbers, $m_{H}$ and $m_{D}$, the dipolar coupling introduces admixtures of other states. This dipole coupling causes a fraction of the states with $\left(m_{H}, m_{D} \pm 1\right)$ to be added to the states $\left(\mathrm{m}_{\mathrm{H}}, \mathrm{m}_{\mathrm{D}}\right)$. By inducing these 'forbidden" transitions, the polarization of the proton can be transferred to the deuteron. At a fixed value of the external magnetic field, $B_{0}$, the transitions $\mathbf{b} \leftrightarrow \mathbf{d}$ and $\mathbf{c} \leftrightarrow \mathbf{e}$ occur at a lower frequency than the $\mathbf{a} \leftrightarrow \mathbf{e}$ and $\mathbf{b} \leftrightarrow \mathbf{f}$ transitions. Thus, by proper choice of the Rf-field frequency, one can select the direction to induce these transitions. By sweeping through the resonant frequency, the populations of the affected states can be inverted. Using this adiabatic fast passage technique, polarization can be transferred from the hydrogen to the deuterium. 


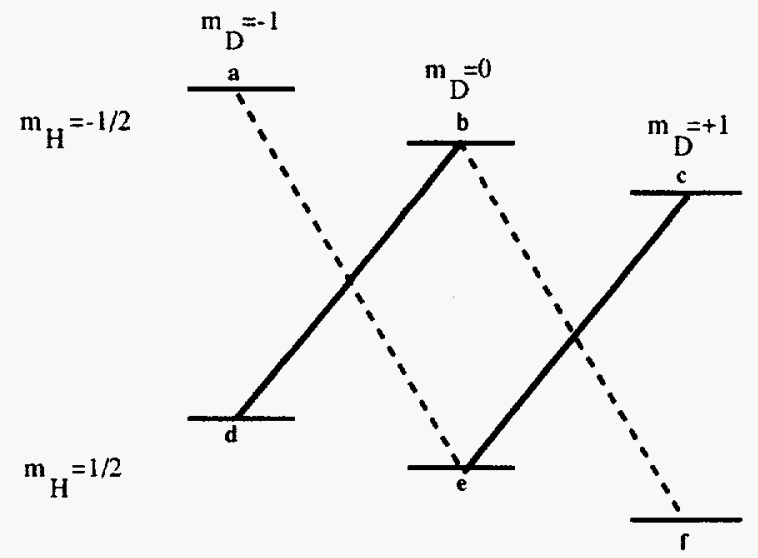

Figure 6. Magnetic energy levels of dipolar coupled $H$ and $D$ nuclei on neighboring HD molecules. "Forbidden" transitions are shown by lines connecting states.

Since the transition rate is proportional to the strength of the Rf-field, $B_{1}$, the latter can be chosen so that the forbidden transitions can be saturated in less than 1 minute. Reducing the external field $B_{0}$ to about $0.03 T$ increases the field uniformity and shifts the NMR frequencies to a convenient range. Since the spin-lattice relaxation time is already $>0.5 \mathrm{hr}$ even at this modest field, there is no significant loss in polarization. The adiabatic fast passage technique is now employed to invert the populations of the appropriate magnetic substates.

In this adiabatic fast passage method, a maximum of $66 \%$ of the polarization of the protons can be transfered to the deuterons. Presently, the efficiency for this process is about $50 \%$, reducing the polarization transfer to $33 \%$ of the hydrogen value. After this, the protons can be repolarized by increasing the field to $17 \mathrm{~T}$ once again. Since the time needed to achieve maximum proton polarization is relatively short compared with the 6.25 day $\mathrm{o}-\mathrm{H}_{2}$ decay time, the deuteron polarization may be built up to a higher level by repeating the entire adiabatic fast passage and proton repolarization procedure again. Once this has been completed, the sample must be held at high field and low temperature until the $\mathrm{o}-\mathrm{H}_{2}$ impurity has decayed and the relaxation time increased. The time-line for this procedure is shown below in figure 7. The upper panel gives $C_{1}^{H}$, the concentration of the magnetically active o- $\mathrm{H}_{2}$ impurity. Adiabatic Fast Passage and proton repolarization take place at day 2.5 and day 6 . The resulting $\mathrm{H}$ and $\mathrm{D}$ polarizations are indicated on the lower panel.

In this way it is possible to make frozen-spin HD ice targets with $80 \%$ free-proton polarization and at least $50 \%$ deuteron vector polarization. (The corresponding deuteron tensor polarization is $20 \%$.)

Because hydrogen and deuterium are polarized separately, one can choose to polarize both as shown in figure 7 or only one or the other. If only polarized $\mathrm{H}$ is desired, then the Adiabatic Fast Passage is omitted. If only polarized $D$ is required, then the repolarization of the hydrogen after the transfer to deterium is omitted. 


\section{SPHICE fabrication and operation}

Small polarized HD samples have been routinely manufactured by A. Honig and his group at Syracuse University for use at the Rochester Laser Fusion facility. A recently completed feasibility study has established a clear path for constructing the larger samples $(2.3 \mathrm{~cm}$ diameter $\times 7.0 \mathrm{~cm}$ in length) required for use at LEGS. Because of the expertise of

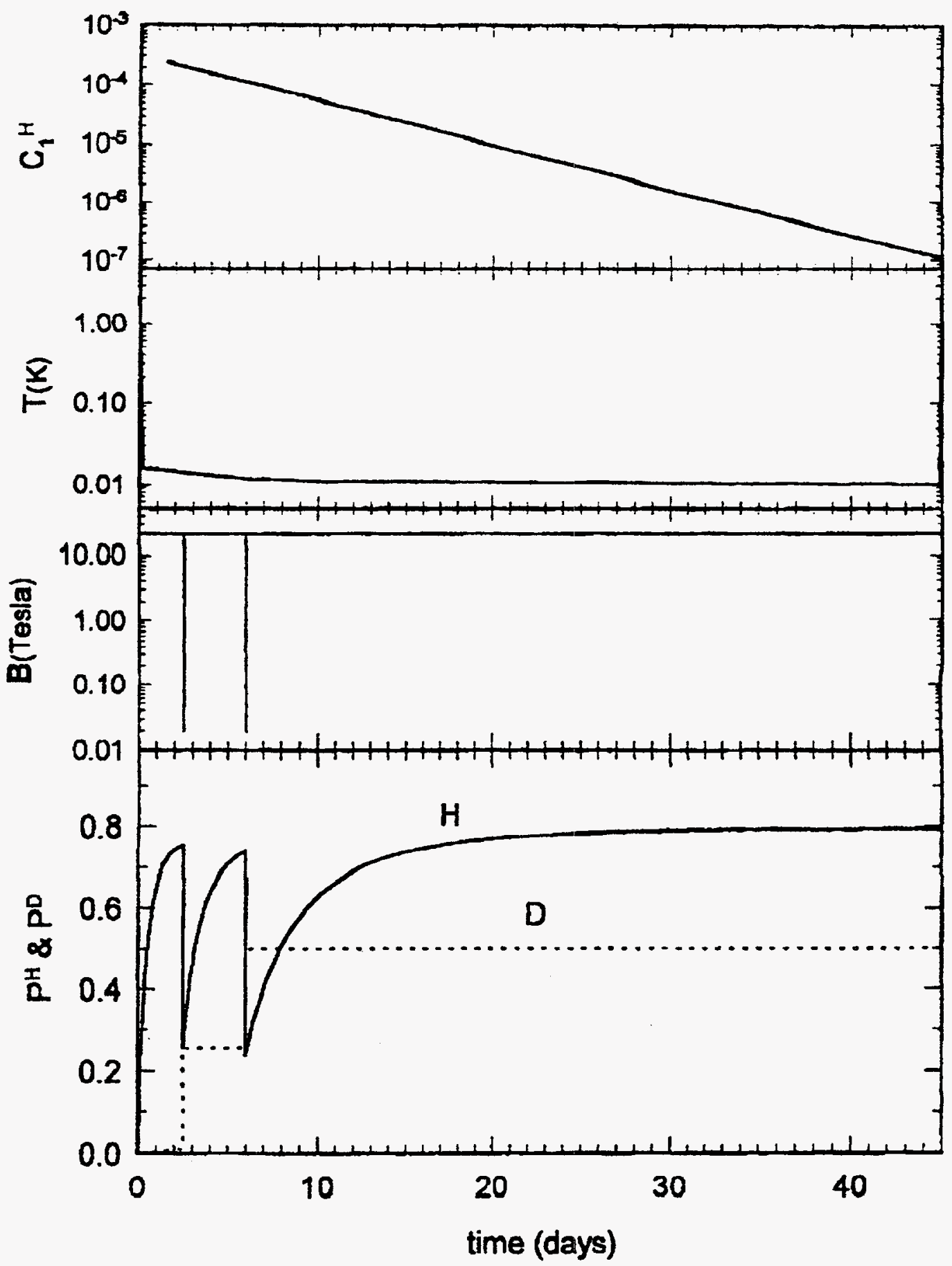

Figure 7. The time-line for polarizing the SPHICE target. $C_{1}^{H}$ (upper panel) is the concentration of $\mathrm{I}=1$ ortho- $\mathrm{H}_{2}$. The $\mathrm{H}$-polarization continuously increases as the $\mathrm{o}-\mathrm{H}_{2}$ concentration decreases. At day 2.5 and day 6 polarization transfer from $\mathrm{H} \rightarrow \mathrm{D}$ is induced by "forbidden" Rf-transitions. The resulting $\mathrm{H}$ and $\mathrm{D}$ polarizations are shown as the solid and dotted curves in the lower panel. 
Honig's group, the targets will initially be manufactured in Syracuse. The cycle time needed to reach $80 \%$ proton polarization and $50 \%$ deuteron polarization, and to complete the $\mathrm{o}-\mathrm{H}_{2} \rightarrow \mathrm{p}-\mathrm{H}_{2}$ relaxation switch, is about 40 to 50 days. In fact, 3 to 4 such $2.3 \mathrm{~cm} \varnothing \times 7.0$ $\mathrm{cm}$ targets, arranged in tandem in the dilution-refrigerator/polarizing-magnet dewar, can be manufactured in almost the same time period. This takes advantage of the fact that high magnetic field uniformity is only critical during the $\mathrm{H} \rightarrow \mathrm{D}$ Adiabatic Fast Passage. Since this proceedure is quite rapid, the position of the the target samples can be adjusted as needed within an extended magnet in which a high field uniformity is maintained over only a portion of the full length.

A key technological step that makes such a target practical for a scattering experiment is the extraction dewar ${ }^{(\mathrm{Ho} 93)}$. This is a $\mathrm{LHe} / \mathrm{LN}_{2}$ dewar that one can insert into the dilution refrigerator, connect to the polarized target, remove it and place it in a low field $\left(\sim 1\right.$ Tesla), comparatively warm $\left(1.5^{\circ} \mathrm{K}\right)$ environment for experimental use. This separates the polarization facility from the experiment and permits the use of a much simpler and less obstructive in-beam dewar than is used with other types of polarized targets.

Once a target has completed the $\sim 45$ day polarizing cycle, it may be transfered to a $1.5^{\circ} \mathrm{K}$ LHe-pumped dewar. Using a suitably aged sample, the relaxation times have been measured at this temperature as a function of holding field during the feasibility study. Most of the experiments used HD samples in long glass tubes with a reservoir to contain

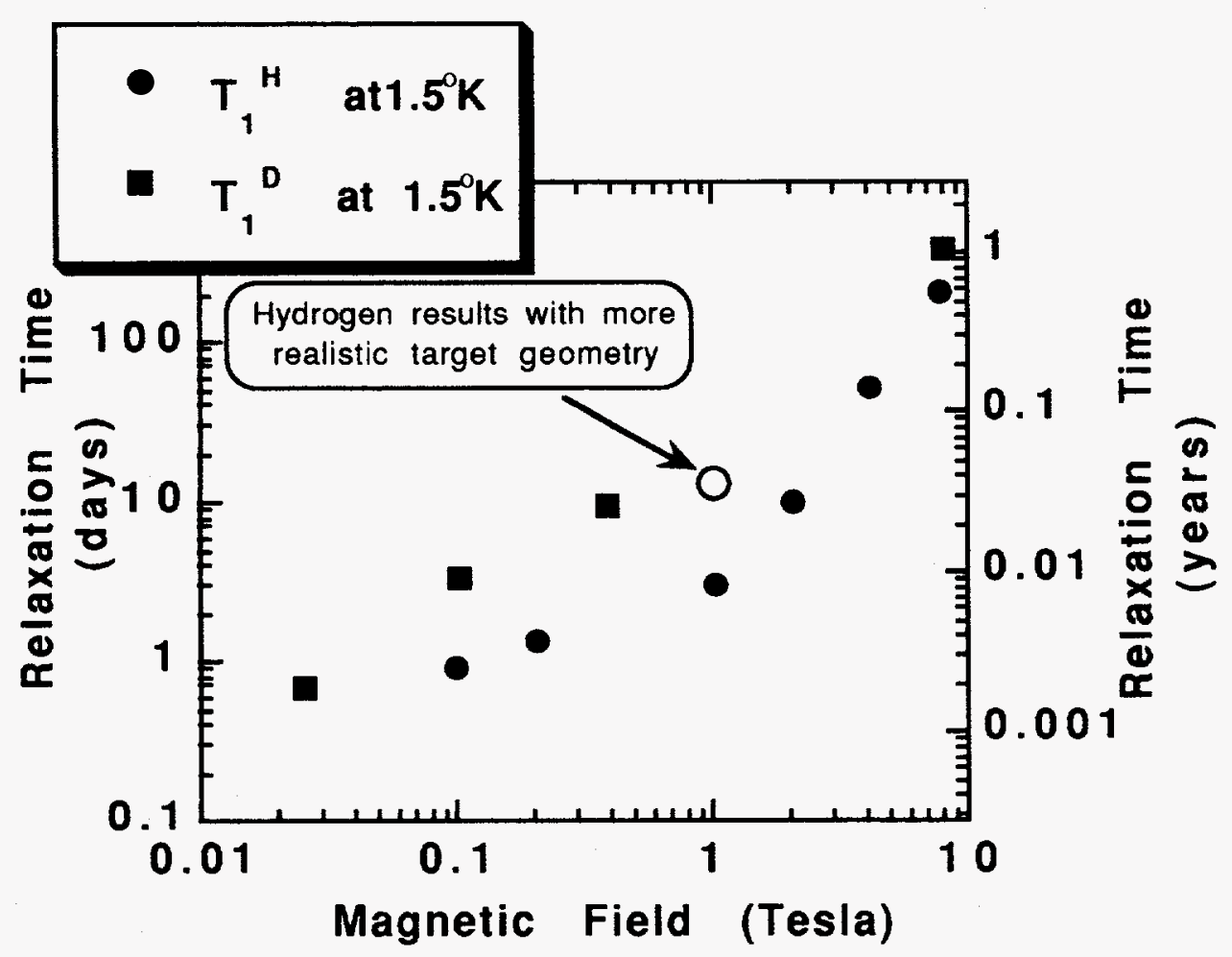

Figure 8. The hydrogen and deuterium relaxation times at $1.5^{\circ} \mathrm{K}$, after a $\sim 45$ day polarizing cycle. (From measurements on HD samples made during the 1993 feasibility study.) 
the HD in the gas phase. (This storage vessel made the samples easy to transfer in and out of a variable temperature dewar.) These "open geometry" results are indicated with the filled symbols in figure 8 . As seen there, the relaxation times for deuterium are always significantly longer than for hydrogen. Subsequent measurements were done in a dilution refrigerator with a closed target geometry more closely approximating that to be used for the production target. The result at $1 \mathrm{~T}$ is shown as the open circle in figure 8 , indicating that a 13 day relaxation time for hydrogen can be achieved at this field. (The corresponding deuterium relaxation time for these conditions is greater than three weeks.) Further investigations of the temperature dependence at $1 \mathrm{~T}$ have found that $T_{1}^{H}$ increases by $20 \%$ at $1.2^{\circ} \mathrm{K}$ and, at $0.45^{\circ} \mathrm{K}, T_{1}^{H}$ increases by more than a factor of two over the $1.5^{\circ} \mathrm{K}$ value. If targets are changed each week, lowering the in-beam temperature from $1.5^{\circ} \mathrm{K}$ to $0.45^{\circ} \mathrm{K}$ raises the average polarization during a measurement from $78 \%$ to more than $88 \%$ of the initial value. To take advantage of this temperature dependence, the in-beam dewar will operate throughout the range from $0.4^{\circ} \mathrm{K}$ to $1.5^{\circ} \mathrm{K}$, with startup experiments using $1.5^{\circ} \mathrm{K}$ operation for simplicity.

At $8 \mathrm{~T}$, the relaxation time is over 250 days for polarized $\mathrm{H}$ and in excess of a year for polarized $\mathrm{D}$. This makes storage and transport of the target possible. Using a storage dewar equiped with an $8 \mathrm{~T}$ magnet, the samples will be brought to BNL. During the experimental running periods, the targets will be transfered, one at a time as needed, from the $8 \mathrm{~T}$ storage vessel to the in-beam dewar. In the in-beam position, the polarizations of the $\mathrm{H}$ and the $\mathrm{D}$ may be oriented independently. (Rotating the holding field will flip the $\mathrm{HD}$ pair and Rf-induced transitions can be used to separately orient the $\mathrm{H}$ polarization.) By holding the target at $1 \mathrm{~T}$ and at temperatures between $0.4^{\circ} \mathrm{K}$ and $1.5^{\circ} \mathrm{K}$, the 3 to 4 samples will provide useable polarized targets in the in-beam configuration for periods greater than their preparation cycle. Use of the higher-field storage dewar between running periods will ensure the full potential of in-beam use, even though the elapsed time may be considerably longer.

After target fabrication has reached a routine phase, the dilution refrigerator, highfield polarizing magnet and all associated target fabrication equipment will be moved to the LEGS facility at BNL.

\section{Advantages over conventional targets}

The experimental quality factors for a polarized target are proportional to (f $\left.\mathrm{P}_{\mathrm{T}}\right)^{2}$, where $f$ is the useful fraction of the species that is polarized to the degree $P_{T}$. One-half of all protons in the target are usefully polarized making SPHICE almost a factor of three better than $\mathrm{NH}_{3}$ targets $(\mathrm{Br} 77)$. As a neutron target, SPHICE represents an even more dramatic improvement because all the neutrons in the target are polarized. The HD target has a quality factor that is more than an order of magnitude larger than $\mathrm{ND}_{3}{ }^{(\mathrm{Br} 77)}$. Although polarized ${ }^{3} \mathrm{He}$ targets offer a reasonable alternative to HD ice, since there is only one neutron in the target and it is polarized, the relative target densities strongly favor SPHICE for use in a continuous beam such as LEGS. (In addition, the higher deuteron tensor polarization available for the SHPICE target offers substantial improvements over existing targets.) 
The $\mathrm{H}$ and $\mathrm{D}$ polarizations that can be expected with SPHICE are compared to the performance of conventional ammonia and butanol targets $(\mathrm{Bo91}, \mathrm{Cr} 90, \mathrm{Me} 88)$ in figure 9 . For temperatures below $0.3 \mathrm{~K}$ where the highest polarizations are achieved with dynamically polarized targets, a ${ }^{3} \mathrm{He}-{ }^{4} \mathrm{He}$ dilution refrigerator is required. At the warmer temperatures, pumping on ${ }^{4} \mathrm{He}$ is sufficient. In contrast to this behavior, the polarization of the SPHICE target is independent of temperature. For SPHICE, only the relaxation time changes with temperature. In addition, as seen in figure 9, high polarizations with ammonia or butanol require $2.5-5.0$ Tesla holding fields. The substantially lower holding fields needed with SPHICE are particularly important in making measurements near thresholds where reaction products are low in energy. Furthermore, the smaller fields means that the coils required are less obtrusive, which is important for large solid angle detector usage.

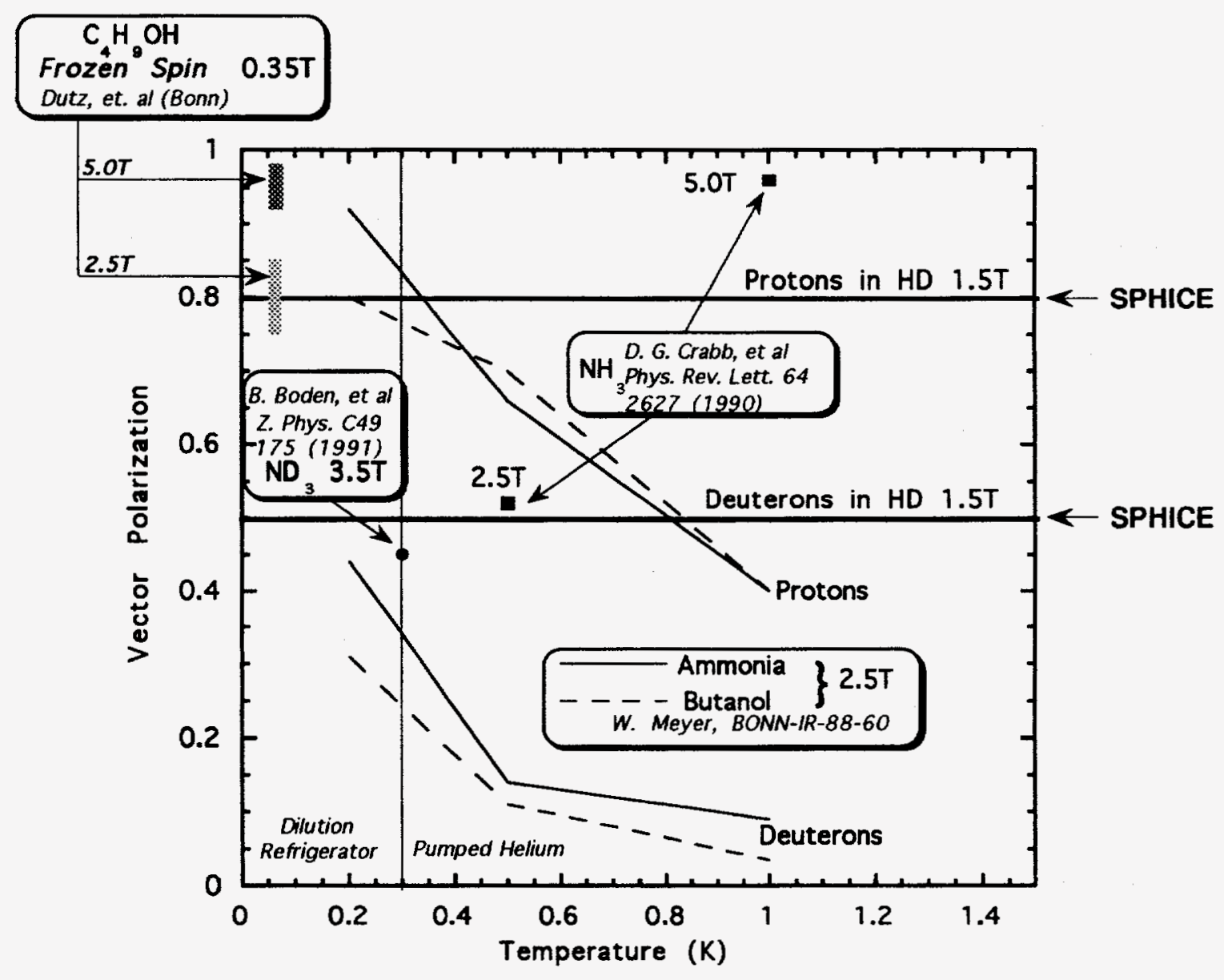

Figure 9. The proton and deuteron vector polarizations that have been achieved using dynamically polarized ammonia and butanol compared to what is expected for the SPHICE target. In this target, the polarizations are independent of the temperature throughout this range, only the relaxation times change. 
Frozen spin butanol targets can also be constructed that provide proton polarizations near $100 \%$. However, this requires temperatures near $60 \mathrm{mK}$ to achieve relaxation times of 130 hours. With these relaxation times, the high field magnet necessary to repolarize the target must also be present while the target is in the beam. Although the in-beam relaxation times obtained for hydrogen at present are of the same order, the SPHICE target offers a tremendous advantage by separating the polarization and experimental facilities.

One complication that arises in SPHICE is due to the low thermal conductivity of HD. The relaxation of the magnetically active $\mathrm{o}-\mathrm{H}_{2}$ to the inert $\mathrm{p}-\mathrm{H}_{2}$ generates more heat than the bulk conductivity of solid HD can carry away. To preserve a uniform temperature throughout the polarizing cycle, the volume of the target cells is threaded with thin $(\leq 25 \mu \mathrm{m})$ aluminum wires. The number of nucleons in Al nuclei is $14 \%(7 \%)$ of the number of hydrogen (deuterium) nuclei, and represents a background that must be subtracted. Nonetheless, this contamination is still a factor of 5 less than that due to nitrogen in ammonia targets.

An additional problem that has been encountered with ammonia targets is the partial polarization of the nitrogen, which affects even asymmetry measurements. The nitrogen polarization is typically $15-20 \%$, and for reactions from $\mathrm{ND}_{3}$, one must subtract data taken with a polarized $\mathrm{NH}_{3}$ target. The aluminum is not polarized at the in-beam conditions of the SPHICE target, simplifying the empty subtraction procedure.

When focusing on measurements from polarized-H (or D) the presence of deuterons (or protons) does not represent any additional complications since the reactions from these light nuclei can be made kinematically complete. In any case, the $H$ and D polarizations can be independently reversed, which makes it possible to simultaneously collect data with both polarizations of the "background" D (or $\mathrm{H}$ ) for a given "target" $\mathrm{H}$ (or D) polarization. Additionally, using $\mathrm{Rf}$ techniques, the $\mathrm{H}$ and $\mathrm{D}$ polariations can be reversed with respect to the holding field, making it possible to explore all beam polarization/ target polarization/holding field combinations for reducing systematic errors.

Because the HD ice target contains only hydrogen and deuterium, much smaller backgrounds are expected for many reactions, making it possible to measure not only asymmetries but absolute cross sections. At present there are no absolute cross section data taken with polarized hydrogen or deuterium targets in real photon beams. SPHICE will provide the first opportunity, and the added feature that $H$ and $D$ measurements can be made simultaneously will lead to a minimization of systematic uncertainties. This is crucial since the key physics questions require a determination of helicity cross sections for the proton-neutron difference. 


\section{LEGS: Energy Range of Measurements with Circularly Polarized Beams}

An inherent difficulty with sum rule measurements is the large energy range over which data must be integrated. Although other proposals exist to measure the DHG sum rule, the polarization and energy-span requirements preclude complete coverage at any one laboratory. Thus, it will always be necessary to combine data from different facilities to get a complete determination of the DHG sum rule. On the other hand, the $1 / \omega^{3}$ weighting of the spin-polarizability integral ensures a more rapid convergence and hence a significantly larger coverage in any one experiment. The fraction of the total $\gamma$ and DHG integrals exhausted as a function of the energy limit of integration has been estimated using the predictions of the VPI-FA93 multipoles(WA93). These are shown in figure 10.

The energy weighting of the $\gamma$ and DHG integrals, combined with the stretched spinconfiguration of the $\Delta$, amplifies the importance of the region of the first baryon resonance. The measurements at LEGS will cover this region, from $\pi$ threshold to 470 $\mathrm{MeV}$. The results of the calculation shown in figure 10 for the operating modes of LEGS are listed in Table 3. These include the newly approved mode of NSLS Ring Operations at $2.8 \mathrm{GeV}$, and the use of $263 \mathrm{~nm}$ light from a new frequency-quadrupled Nd-YLF laser that is currently being developed in a joint FRASCATI-BNL program.

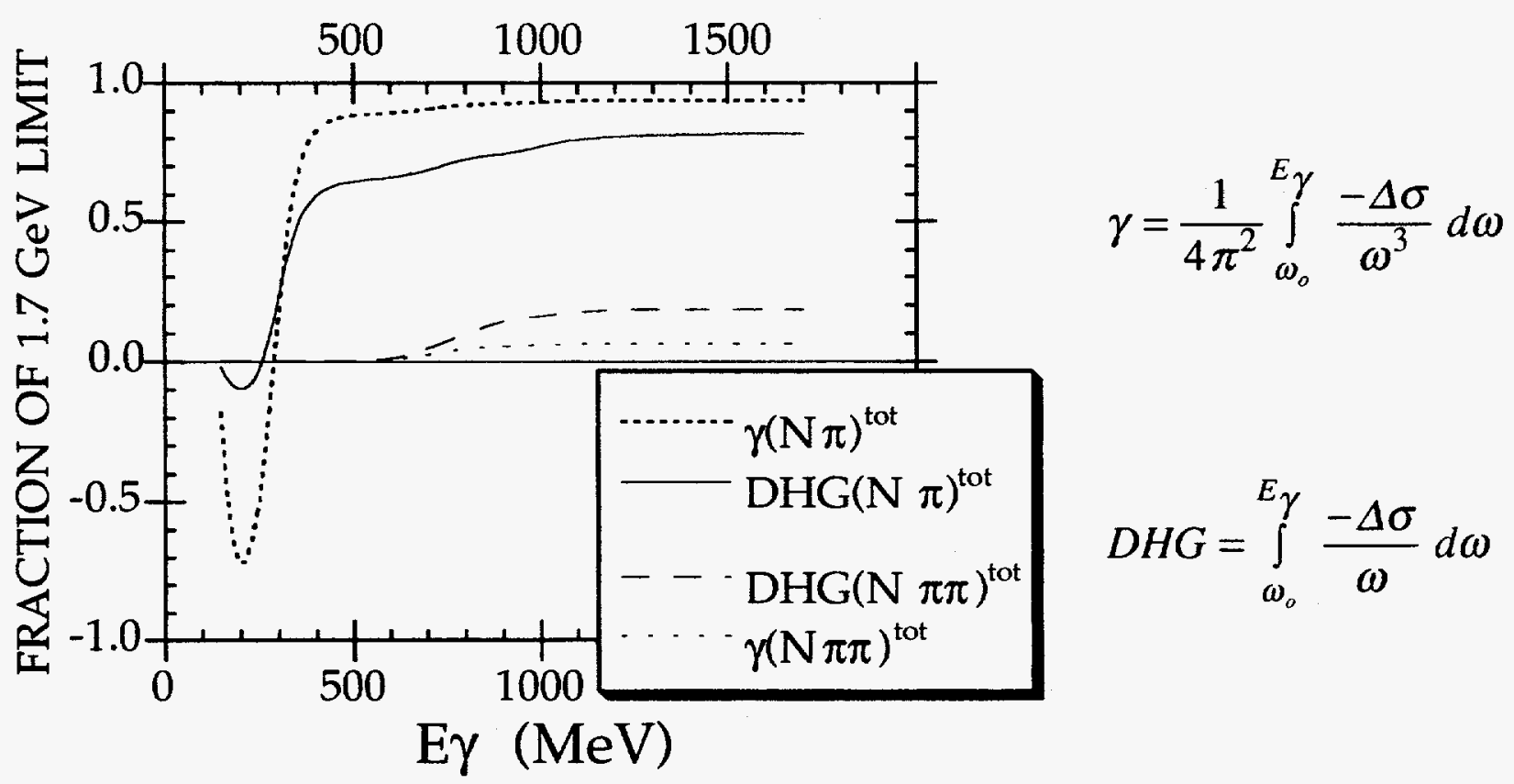

Figure 10. The fraction of the spin-dependent polarizability and DHG integrals of $\Delta \sigma=\left(\sigma_{3 / 2}-\sigma_{1 / 2}\right)$, as a function of the upper limit of integration, compared with the values computed up to $1.7 \mathrm{GeV}$ (SWK94), using the VPI-FA93 amplitudes(WA93). The 'Total' value, the sum of the three isospin contributions (VV, VS, and SS), represent the expected results for the proton. 
Table 3. Fraction of the spin-dependent polarizability, $\gamma$, and the DHG integrals, compared to their values at $1.7 \mathrm{GeV}$ as computed from the amplitudes of FA93(WA93), for different LEGS operating modes. Here, $\lambda$ is the wavelength of the laser, $E_{e}$ is the energy of the stored electron beam, and $E_{\gamma}$ is the maximum LEGS $\gamma$-ray beam energy.

\begin{tabular}{|ccccc|}
\hline $\begin{array}{c}\lambda \\
(\mathrm{nm})\end{array}$ & $\begin{array}{c}\mathrm{E}_{\mathrm{e}} \\
(\mathrm{GeV})\end{array}$ & $\begin{array}{c}\mathrm{E}_{\gamma}(\mathrm{MAX}) \\
(\mathrm{MeV})\end{array}$ & $\begin{array}{c}\text { Fraction of } 1.7 \mathrm{GeV} \text { value } \\
\gamma \text { integral }\end{array}$ & DHG integral \\
\hline 333 & 2.584 & 332 & 0.54 & 0.41 \\
300 & 2.584 & 363 & 0.74 & 0.53 \\
300 & 2.800 & 422 & 0.86 & 0.61 \\
263 & 2.800 & 471 & 0.88 & 0.64 \\
\hline
\end{tabular}

With the present electron orbit, there is a low-energy tagging limit of $185 \mathrm{MeV}$, and the full range from 185 to $471 \mathrm{MeV}$ will be covered during Standard Operations of the storage ring. For the measurements between $\pi$-threshold and $185 \mathrm{MeV}$, a local-bump in the standard orbit will be used to modify the acceptance of the tagging spectrometer. Although accelerator-studies are required to develope this bump, the NSLS accelerator staff foresee no complications, and user mechanisms are already in place to schedule running in such special conditions.

Almost $90 \%$ of the $\gamma$ integral will be covered by this set of data, providing a reasonable comparison with the $\chi \mathrm{PT}$ predictions. These proton-neutron difference measurements will also cover about $2 / 3$ of the DHG integral and provide the first opportunity of testing the isospin decompositions that have been proposed by multipole analyses. For the DHG sum rule to correctly account for the isospin-splitting of the nucleon, the VS component, $D H G_{V S}=1 / 2\left(D H G_{p}-D H G_{n}\right)$, would have to be dramatically different from the decomposition shown in figure 4 .

Ultimately, a complete test of the DHG sum rule will require combining these measurements with higher energy data from another laboratory. At present, higher energy experiments are in preparation at Bonn, CEBAF and Mainz using polarized bremsstrahlung beams incident on polarized protons (neutrons) in $\mathrm{NH}_{3}\left(\mathrm{ND}_{3}\right)$ targets. Higher energy DHG measurements using laser-backscattered photons are also planned for the GRAAL facility now under construction in Grenoble, and a laser-backscattering facility is currently under study for CEBAF. Beam-polarization experiments are characterized by a quality factor of $\left(\mathrm{P}_{\gamma}\right)^{2}$, where $\mathrm{P}_{\gamma}$ is the $\gamma$-ray polarization. Laser-backscattered beams produce circular polarizations of $\approx 100 \%$, while $\approx 50 \%$ can be achieved with a bremsstrahlung beam. The backscattered beam thus represents an improvement of a factor of 4 over a bremsstrahlung beam. When this is combined with the $\left(f \mathrm{P}_{\mathrm{T}}\right)^{2}$ quality factor for the polarized target discussed in the previous section, the resulting enhancement of the backscattered-beam-\&-SPHICE-target over that of the bremsstrahlung-\&-ammonia-target combination becomes an order of magnitude for measurements on protons and more than a factor of 50 for neutrons. These represent dramatic potential gains in data quality. 


\section{Detector Array: Measurements of the Polarized Cross Sections}

\section{SASY - The Spin ASYmmetry Detector Array}

The Spin ASYmmetry (SASY) array, a high efficiency, large solid angle detector for measuring total reaction cross sections, will be constructed to study reactions of polarized photons on polarized protons, neutrons and deuterons. As shown in figure 11, this detector will consist of several "layers" to provide complete determination of angle, energy, and particle identity for all reactions induced by photons on hydrogen and deuterium over the entire energy range planned for LEGS (incident $\gamma$-ray energies up to $470 \mathrm{MeV}$ ). The principal motivations for assembling this array are to measure the total reaction cross sections needed in the determination of the spin-dependent polarizability of the nucleon and Drell-Hearn-Gerasimov sum rule. However, the ability of this detector to completely identify all reaction channels will allow angular distributions to be measured separately for each individual channel with target-spin and beam-helicity defined. Such

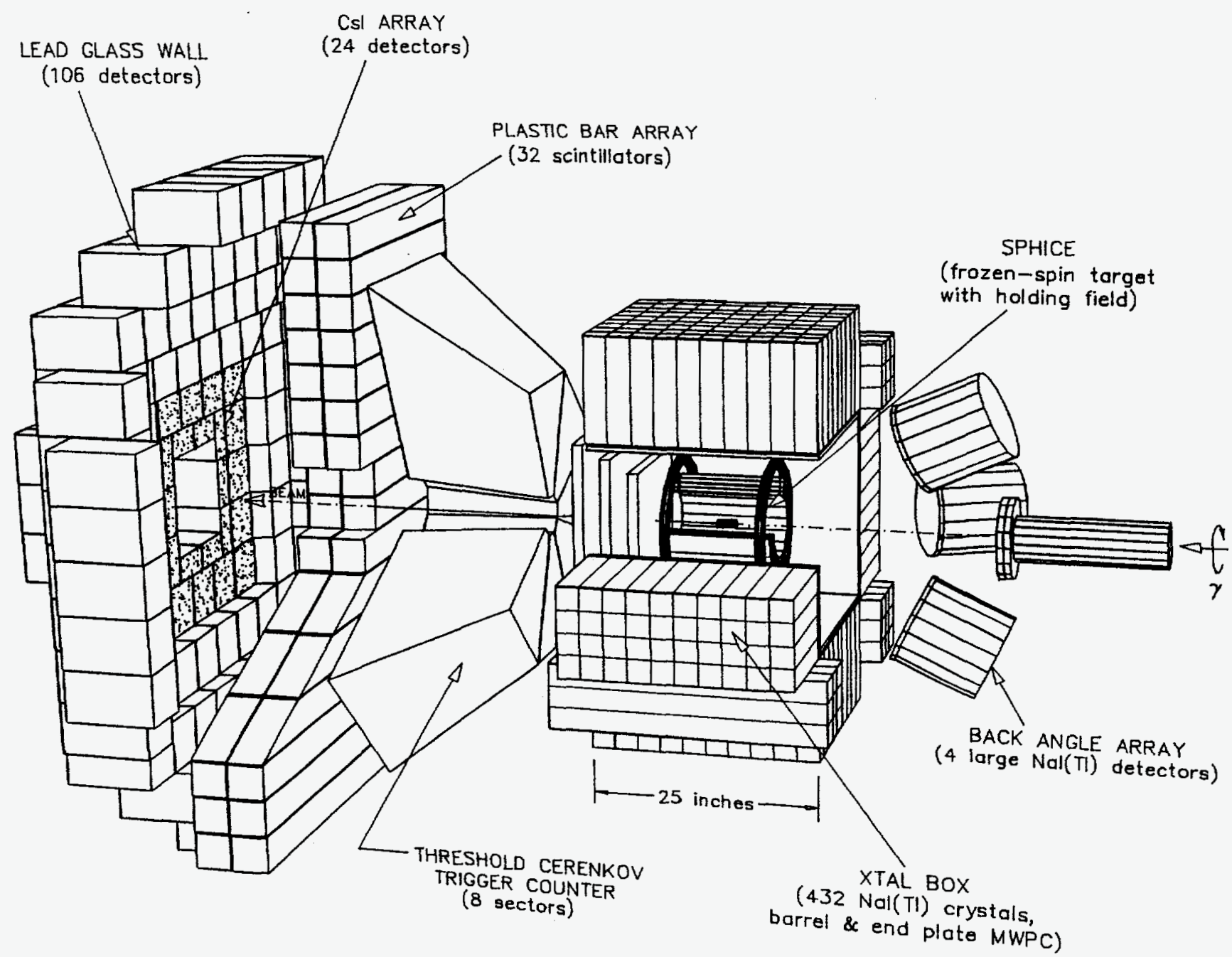

Figure 11. Cutaway view of the Spin ASYmmetry (SASY) array - the XTAL BOX, with wire chambers and an end-cap of plastic, CsI and Pb-glass detectors surrounding the SPHICE target and its holding field. Atomic electrons are suppressed by the threshold Cerenkov counter. 
measurements will provide important new constraints on photoproduction amplitudes.

The SASY array will consist of several subsystems of wire chambers, scintillators and Cerenkov detectors. At the center of the detector, the SPHICE target will be surrounded by cylindrical wire chambers inside a pair of superconducting Helmholtz-like coils with a diameter of $32 \mathrm{~cm}$. These coils will produce a field of 1 Tesla at the target, with good uniformity, to maintain its polarization. The cylindrical wire chambers will cover scattering angles from 40 to 140 degrees. Track reconstruction in the axial magnetic field will determine the sign of charged pions and provide a momentum measurement for pions and low energy protons (up to about $70 \mathrm{MeV}$ ). A plastic scintillator box surrounding these chambers, included in the event trigger, will provide energy-loss measurement to identify charged particles. Around this entire assembly will be the XTAL BOX(Wi88), containing $432 \mathrm{NaI}(\mathrm{Tl})$ crystals, which will serve as a calorimeter for charged particles as well as gamma-rays from scattering and from neutral-pion production. The acceptance of the cylindrical wire chambers is matched to the XTAL BOX which subtends angles from 40 to 140 degrees in scattering angle.

At the open front (small reaction angle) end of the XTAL BOX, a stack of large area detectors will measure the higher energy, forward reaction products, extending the coverage from $30^{\circ}$ to about $5^{\circ}$. (The region between the $30^{\circ}$ limit of this forward end-cap and the $40^{\circ}$ minimum acceptance of the XTAL BOX is shadowed by the Helmholtz coils and their supports.) In the first layer of this end-cap, three planes of multi-wire proportional chambers, located in the external field of the Helmholtz coils and just inside the XTAL BOX, will track charged pions and protons and distinguish them from electrons. The requirement that a particle produce a track with radius of curvature consistent with its energy (measured by downstream stopping detectors) discriminates strongly against electrons, which scatter significantly in the wire chamber gas and foils. (The high efficiency of this method of electron rejection by track reconstruction has already been demonstrated in LEGS Exps. L5, 7 and 8.) Although this rejection technique is quite effective, it requires off-line analysis. The magnetic field will bend almost all electrons into the forward end-cap, but the potentially large numbers of these atomic triggers will be suppressed by a segmented, threshold gas-Cerenkov counter located immediately behind the planer multi-wire chambers. At a distance of $140 \mathrm{~cm}$ from the target, an array of plastic scintillator bars, $160 \mathrm{~cm}$ by $160 \mathrm{~cm}$ by $20 \mathrm{~cm}$ thick, will detect protons, pions, and neutrons. For single-pion production channels, these bars cover most of the angular range for recoiling nucleons. Energy, at least for the lower part of the range, can be determined by time-of-flight for all particles and by light output for charged particles to better than 3\%. At small angles, protons from $\pi^{\mathrm{o}}$-production and from deuteron photodisintegration punch through the bar array. These are stopped in an array of intrinsic CsI detectors. These crystals have excellent time and energy resolution for charged particles as well as photons. Behind the scintillator bars, and around the CsI array, a segmented wall of lead glass blocks will determine photon (and therefore $\pi^{\circ}$ ) energies and angles.

At backward angles, the opening in the crystal box will be sampled by 4 moderately large $(25 \mathrm{~cm} \times 30 \mathrm{~cm}) \mathrm{NaI}$ detectors. These will detect and identify photons as well as low energy pions and protons. The coverage here is not as complete as in the forward direction since room is required for the in-beam target dewar. 
Beyond holding the spin of the SPHICE target, the magnetic fields of the Helmholtz coils in SASY are used mainly to determine the sign of charged pions. Particle-ID and energy measurement rely heavily on energy loss and deposition. The ultimate limitation on this approach is the accuracy of corrections that have to be made to account for reactionlosses of high-energy particles in scintillators. At high-beam energies (approximately 500 $\mathrm{MeV}$ and above), these corrections can become quite large and magnetic analysis must be relied upon more heavily. However, the SASY array is quite well matched to the energy range of the planned measurements at LEGS. The ability of this detector to measure absolute cross sections, combined with its high symmetry, will allow consistency checks to be made, which will be quite important in minimizing the uncertainties in reaction-loss corrections. In particular, summing over target spin orientations with linearly polarized photons provides data to be compared with the cross sections and asymmetries that have been previously measured at LEGS in this energy region ${ }^{(\mathrm{B} 191, \mathrm{B19})}$. Also, beam polarization asymmetries can be measured with any orientation of the reaction plane and compared with each other. Such measurements will be important in determining the absolute efficiency of this complex device. The precision results that will be obtained from this detector make it an appropriate complement to the high quality of both the LEGS beam and the SPHICE target.

The design of this detector is particularly attractive because many of its components are in-hand. The XTAL BOX, serving as the central calorimeter, has already been mounted on the LEGS beam line. A $10 \mathrm{~cm}$ thick stack of plastic scintillator bars are presently being used in Exp. $L 8$ and a $2^{\text {nd }} 10 \mathrm{~cm}$ thick stack will be purchased for use with the forward angle part of this detector array. The forward-angle $\mathrm{Pb}$-glass array was acquired from an AGS experiment. (About a third of the phototubes will have to be replaced.) The back-angle NaI detectors were used in Exps. L1-8 and are in-hand. The main construction effort associated with this detector is in the construction of the wire chambers.

\section{Monte Carlo Simulations}

Simulations of the response of the SASY array are underway. The large solid angle scintillator coverage is ideally suilted for the detection of $\gamma$-rays. A Monte Carlo code, using GEANT, has already been developed by $M$. Blecher of this collaboration for modeling the XTAL BOX. A simple indicator of the effectiveness of the XTAL BOX for $\gamma$ ray detection is the fraction of the total energy deposited in the active volume. This is listed in table 4 for the range of interest. The energy containment is clearly excellent.

Table 4. Photon stopping efficiency for the XTAL BOX.

\begin{tabular}{|c|c|c|}
$\begin{array}{c}\text { Incident } \\
\text { Energy } \\
(\mathbf{M e V})\end{array}$ & $\begin{array}{c}\text { Energy } \\
\text { Deposited in } \\
\text { NaI XTAL's }\end{array}$ & $\begin{array}{c}\text { Unobserved } \\
\text { Energy } \\
(\boldsymbol{\%})\end{array}$ \\
\hline 500 & 465 & 7 \\
\hline 400 & 373 & 7 \\
\hline 300 & 282 & 6 \\
\hline 200 & 190 & 5 \\
\hline 100 & 96 & 4 \\
\hline
\end{tabular}


Table 5. Final states produced from the SPHICE target below $309 \mathrm{MeV}$.

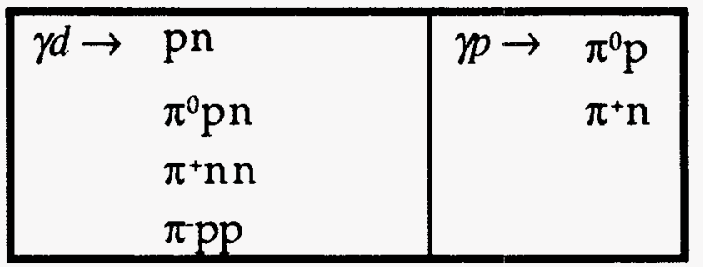

In considering the detection of charged particles, the problem naturally divides into the region below $2 \pi$-threshold ( $309 \mathrm{MeV}$ beam energy), and the region above. The dominant reactions to be considered below $309 \mathrm{MeV}$ are listed in table 5. One of these reactions,
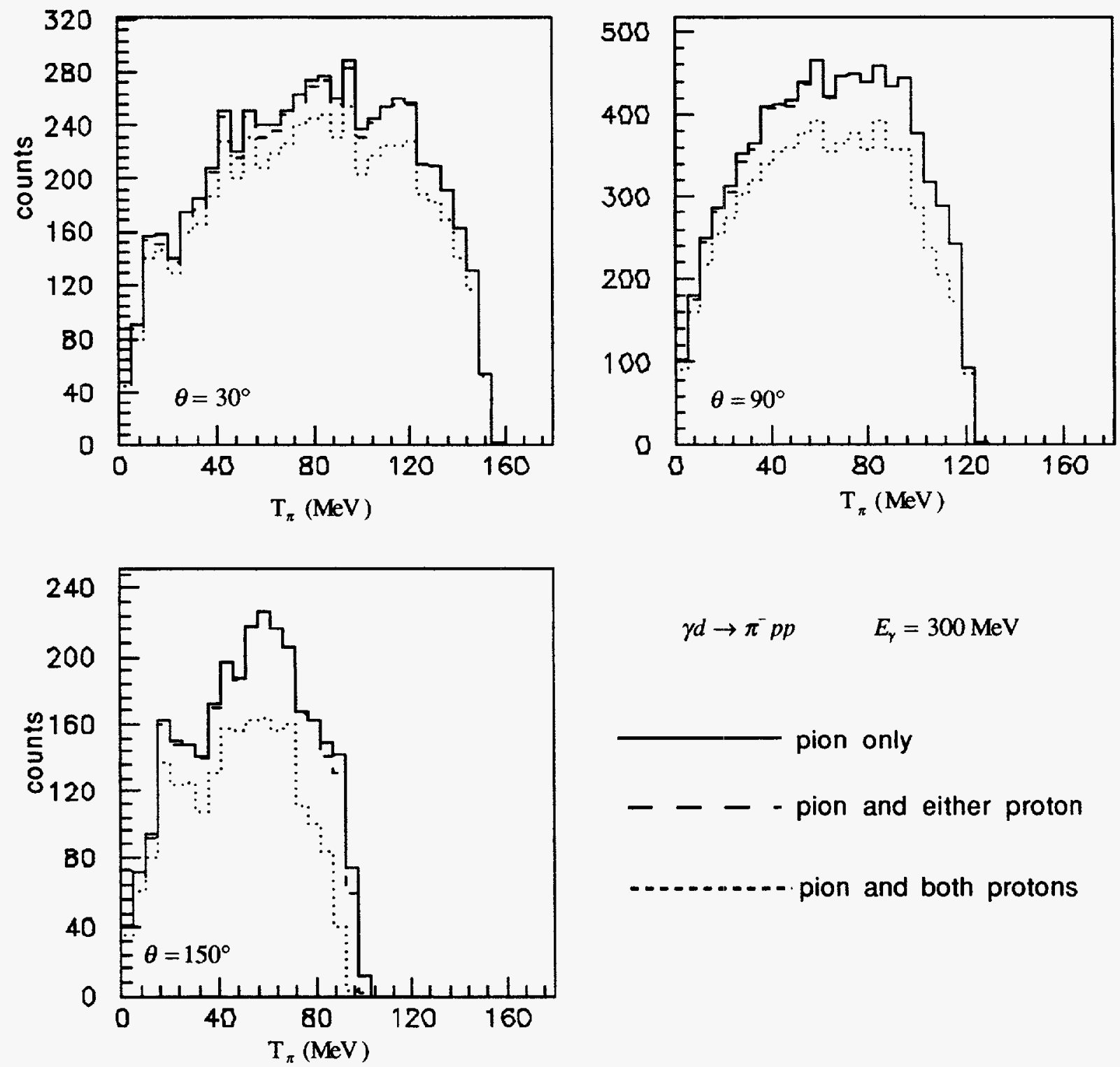

$$
\gamma d \rightarrow \pi^{-} p p \quad E_{\gamma}=300 \mathrm{MeV}
$$

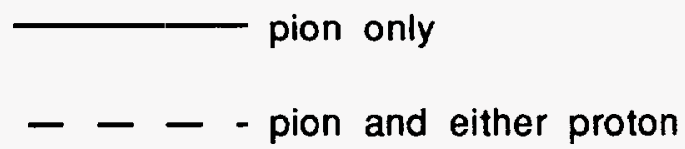

pion and both protons

Figure 12. Pion spectra for the $\gamma d \rightarrow \pi^{-} p p$ reaction. The solid line represents all pions. The long dashed line indicates pions for which either of the two protons are emitted between $20^{\circ}$ and $160^{\circ}$. The short dashed line represents the pions for which both protons are in this angular range. 
$\gamma \mathrm{d} \rightarrow \mathrm{pn}$, may be isolated with a single-arm trigger. The photodisintegration of deuterium is the only source of high energy protons. Measurement of the proton angle and energy along with the photon tag, overdetermines the kinematics for this reaction. These protons are found in a cleanly separated peak, making extraction of yields straightforward. The other final states generally require the detection of at least 2 particles, but the identification of these processes is also straight forward, due to the large solid angle coverage of SASY. For example, consider the $\gamma d \rightarrow \pi^{-} p p$ reaction. The geometrical acceptance of SASY for the detection of $\pi$-singles, $\pi$-p, and $\pi$-pp coincidences from this reaction is shown in figure 12 , assuming that the particles are distributed according to phase space and ignoring the fields of the Helmholtz coils. Detecting any two of the three final particles is sufficient to reconstruct the event, and requiring one proton in coincidence with the pion makes only a small change in the acceptance. Furthermore, the efficiency for detecting all three outgoing particles is quite reasonable (70 to $90 \%$ ), and will provide a valuable cross check of the acceptance.

Certainly, these simple estimates will be modified by the quasi-free contribution to the $\gamma d \rightarrow \pi^{-} p p$ reaction. Nevertheless, the kinematics will not change because of the quasifree contribution, only the distrubutions. Furthermore, accurate trajectory reconstruction in the magnetic field of the Helmholtz coils will still permit reconstruction of these events. Since detecting two particle in the final state determines the third, the ratio of two and three particle acceptances will not change.

At photon energies above the $2 \pi$-threshold, the situation becomes more complicated. Now the list of reactions is expanded to include those listed in table 6.

Table 6. Two pion final states produced from the SPHICE target.

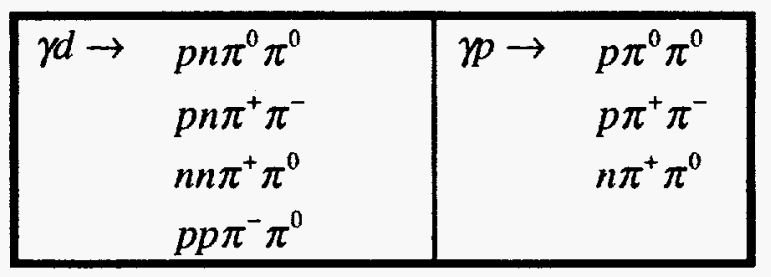

The reactions with three charged particles in the final state: $\gamma d \rightarrow p n \pi^{+} \pi^{-}$, $\gamma d \rightarrow p p \pi^{-} \pi^{0}$ and $\gamma p \rightarrow p \pi^{+} \pi^{-}$, can be readily separated using a technique similar to that described for $\gamma d \rightarrow \pi^{-} p p$. The third of these reactions is complete when either two or three of the particles are detected. The other two reactions will produce an invariant mass that differs from that expected from the $\gamma$-ray energy by a pion mass when all three charged particles are detected.

The $\not p \rightarrow n \pi^{+} \pi^{0}$ and $\gamma d \rightarrow n n \pi^{+} \pi^{0}$ reactions are somewhat more complicated. These require the detection of the $\pi^{+}$and the $\pi^{0}$ in coincidence. Then the $n \pi^{+} \pi^{0}$ events will appear as a peak on the $n n \pi^{+} \pi^{0}$ continuum. For the reactions involving two neutral pions 
in the final state, we rely on the large coverage of the XTAL BOX and the Pb-glass detectors to reconstruct at least three $\gamma$ 's.

Monte Carlo studies are continuing in an effort to optimize the potential of the SASY array to disentangle all possible multi-particle final states. Although this is a complex problem, it must be remembered that the cross sections are dominated by single $\pi$-production throughout the energy range of the planned LEGS measurements ${ }^{(\mathrm{Mu} 93)}$. Such processes are kinematically overdetermined and easily extracted with high efficiency.

\section{Count rate Estimates}

To estimate the yields from reactions on polarized- $\mathrm{H}$ that can be anticipated in these measurements (yields from neutrons in polarized-D follow in exactly the same way), we use the typical LEGS flux of $10^{5} \mathrm{~s}^{-1}$ in a $5 \mathrm{MeV}$ tagging interval of photon energy. The density of the solid HD is $0.15 \mathrm{~g} / \mathrm{cc}$ and the cell length is $7.0 \mathrm{~cm}$. This represents a freeproton thickness of $0.35 \mathrm{~g} / \mathrm{cm}^{2}$. Assuming a coverage in SASY of $80 \%$ of $4 \pi$ and a data acquisition live-time of $80 \%$, the yield in 1 day of running is

$$
N\left(\Delta E_{\gamma}=5 M e V\right)=(1161) \bullet \sigma(\mu b) \quad .
$$

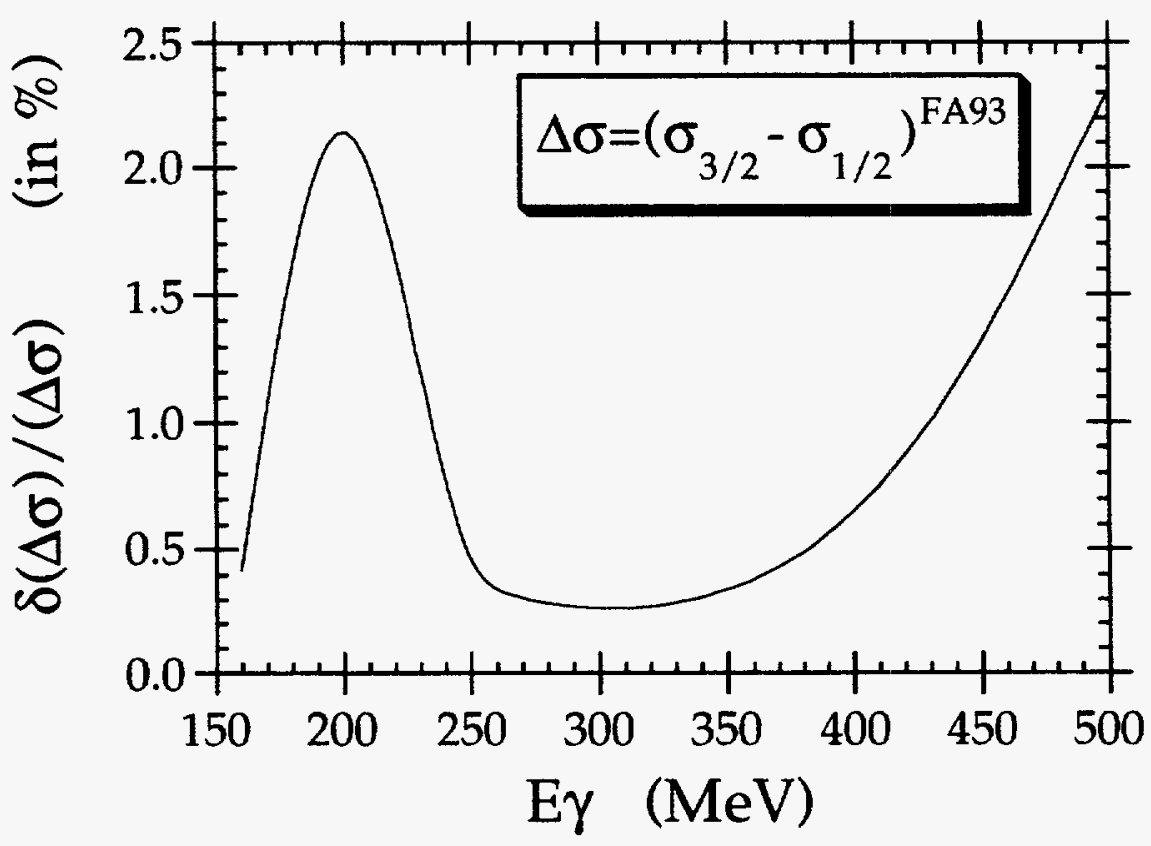

Figure 13. The error (in \%) for the cross section difference $\Delta \sigma=\sigma_{3 / 2}-\sigma_{1 / 2}$, calculated using the FA93 single- $\pi$ production amplitudes ${ }^{(W A 93)}$, as measured by SASY in a $5 \mathrm{MeV}$ tagging interval in one day. 
Thus, for a reaction channel with a total cross section of $1 \mu \mathrm{b}$, a $1 \%$ statistical accuracy can be achieved in 10 days. The dominant reaction channel in the LEGS energy range, $1-\pi$ production, has cross sections that are 2 orders of magnitude larger. The accuracy of these measurements will clearly be dominated by systematic uncertainties.

The FA93 amplitudes have been used to estimate the polarization-difference cross section $\Delta \sigma=\sigma_{3 / 2}-\sigma_{1 / 2}$ that appears in the integrand of both the spin-polarizability and the DHG sum rules. The expected statistical error in $\Delta \sigma$ after a single day of running is shown in figure 13 as a function of beam energy for a beam polarization of $\mathrm{P}_{\gamma}=0.9$ and a proton polarization of $P_{\mathrm{T}}=0.8$. (Here it has been assumed that data are binned by $5 \mathrm{MeV}$, the minimum LEGS beam resolution). Five days of running will reduce these errors below $1 \%$ at all energies.

The overlapping tagging intervals listed in Table 7 will be used to cover the full range of energies available at LEGS. This maintains the high polarization and provides essential cross checks. Five days of full cell, and 2 empty, at each setting amount to about one month of beam on target. The same pair of helicity-1/2 and $3 / 2$ cross sections can be

Table 7. Operating modes that will be used to cover the full energy range.

\begin{tabular}{|cccc|}
\hline $\begin{array}{c}\text { Tagging interval } \\
(\mathrm{MeV})\end{array}$ & $\begin{array}{c}\lambda \mathrm{L} \\
(\mathrm{nm})\end{array}$ & $\begin{array}{c}E_{\mathrm{e}} \\
(\mathrm{GeV})\end{array}$ & Ring Operations \\
\hline $135-236$ & 488 & 2.58 & Special \\
$185-236$ & 488 & 2.58 & Standard \\
$210-332$ & $364-334$ & 2.58 & Standard \\
$285-406$ & 263 & 2.58 & Standard \\
$350-471$ & 263 & 2.80 & Standard \\
\hline
\end{tabular}

measured by flipping either the photon spin or the target spin. Nonetheless, both will be done as a check on systematic uncertainties. This brings the time required for measurements on polarized- $\mathrm{H}$ up to 2 months. The corresponding time required for measurements on the neutron is 5 months, due to the lower deuteron polarization $\left(\mathrm{P}_{\mathrm{T}}=0.5\right)$. These represent months of a full beam-on-target. The combined NSLS-LEGS running efficiency is approximately $2 / 3$, so that this full set of measurements will actually occupy about 10 months. 
References

(Ad93) B. Adeva et al., Phys. Lett. B302, 533 (1993).

(Al84) K.H Althof, et al., Z. Phys. C26, 175 (1984).

(Al89) K. H. Althof, et al., Z. Phys. C43, 375 (1989).

(A191) N. Alexander, J. Barden, Q. Fan and A. Honig, Rev. Sci. Instrum. 62, 2729, (1991).

(Am92) P. Amaudruz et al., Phys. Lett. B295, 159 (1992).

(AIL89) M. Anselmino, Ioffe and Leader, Sov. J. Nucl. Phys. 49, 136(1989).

(An93) P. L. Anthony et al., SLAC preprint, SLAC-PUB-6101 (1993).

(As88) J. Ashman et al., Phys. Lett. B206,364 (1988); Nucl. Phys. B328,1 (1989).

(BDS75) I. Barker, A. Donnachie and J. Storrow, Nucl. Phys. B95, 347 (1975).

(Ba60) A.M. Baldin, Nucl. Phys. 18, 310 (1960).

(BD67)) G. Barton and N. Dombey, Phys. Rev. 162, 1520 (1967).

(B192) V. D. Burkert and B. L. Ioffe, Phys. Lett B296, 223 (1992)

(Bj66) J. D. Björken, Phys. Rev. 148, 1476 (1966); Phys. Rev. D1, 1376 (1971).

(BKKM92) V. Bernard, N. Kaiser, J. Kambor and Ulf-G. Meißner, Nucl. Phys. B388, 315 (1992).

(BKM93) V. Bernard, N. Kaiser and Ulf-G. Meissner, Phys. Rev D48, 3062 (1993).

(BL93) V. Burket and Z. Li, Phy. Rev D47, 46 (1993).

(B191) LEGS Collaboration: G. S. Blanpied, M. Blecher, A. Caracappa, C. Djalali, M-A. Duval, G. Giordano, S. Hoblit, O. C. Kistner, G. Matone, L. Miceli, W. K. Mize, B. M. Preedom, A. M. Sandorfi, C. Schaerf, R. M. Sealock, C.E. Thorn, S. T. Thornton, K. Vaziri, C. S. Whisnant, Phys. Rev. Lett. 67, 1206 (1991).

(B192) LEGS Collaboration: G. Blanpied, M. Blecher, A. Caracappa, C. Djalali, M.-A. Duval, G. Giordano, S. Hoblit, M. Khandaker, O. C. Kistner, G. Matone, L. Miceli, W. K. Mize, B. M. Preedom, A. M. Sandorfi, C. Schaerf, R. M. Sealock, C. E. Thorn, S. T. Thornton, K. Vaziri, C. S. Whisnant, X. Zhao, and M, A. Moinester, Phys. Rev. Lett. 69, 1880 (1992).

(Bo91) B. Boden et al., Z. Phys. C49, 175 (1991). 
(Br77) J. A. Brown, PhD thesis, State University of New York at Stony Brook, 1977, (Unpublished).

(Bu93) V. Burkert, Proc. 6th Int. Workshop on Perspectives in Intermediate Energy Nuclear Physics, Trieste (1993); CEBAF-PR-93-031; extensions of the calculations of (BL93).

(Cr90) D.G. Crabb, et al., Phys. Rev. Lett. 64, 2627 (1990).

(DG70) M. Damashek and F. Gilman, Phys. Rev. D1, 1319 (1970).

(DH66) S. D. Drell and A.C. Hearn, Phys. Rev. Lett. 16, 908 (1966)

(EK93) J. Ellis and M. Karliner, Phys. Lett B313, 131 (1993)

(FF69) G. Fox and D. Freedman, Phys. Rev. 182, 1628 (1969);

H. Abarbanel and M. Goldberger, Phys. Rev. 165, 1594 (1968).

(Ge66) S.B. Gerasimov, Sov. J. Nucl. Phys. 2, 430 (1966).

(GG54) M. Gell-Mann and M. L. Goldberger, Phys. Rev. 96, 1433 (1954).

(GGT54) M. Gell-Mann, M. L. Goldberger and W. Thirring, Phys. Rev. 95, 1433 (1954).

(HM76) A. Honig and H. Mano, Phys. Rev. B14, 1858 (1976).

(Ho67) A. Honig, Phys. Rev. Lett. 19, 1009 (1967).

(Ho89) A. Honig, "Evaporation of Solid Polarized HD and Pure Ortho-D2", Eighth International Symposium on High-Energy Spin Physics, Vol. 2, Minneapolis, $\mathrm{MN}, 1998$. AIP Conference proceedings 189, 1554 (1989).

(Ho89b) A. Honig, N. Alexander and S. Yucel, Workshop on Muon-Catalyzed-Fusion, Sanibel Island, FL, May 2-6, 1988. AIP conference proceedings 181, 199 (1989).

(Ho93) A. Honig, N. Alexander, Q. Fan, X. Wei and Y. Y. Yu, "Achievement of Transportable Polarized D, in Solid HD, With a One Day PassivelyMaintained Polarization", Workshop on Polarized Ion Sources and Polarized Gas Targets, University of Wisconsin, May 23-27, 1993. To be published in AIP conference series.

(Io76) B. L. Ioffe, Phys. Lett. 63B, 425 (1976).

(Ji93) X. Ji, Phys. Lett. B309, 187 (1993);

X. Ji and P. Unran, Phys. Lett. B333, 228 (1994)

(Jo93) C.E. Jones, et al., Phys. Rev. C47, 110 (1993). 
(Ka73) I. Karliner, Phys. Rev. D7, 2717 (1973).

(Ka75) V. Kandansky, Ph.D. thesis (unpublished), BONN-IR-75-54.

(Ki) W. F. Kielhorn, Ph.D. thesis, University of Texas, Austin. Los Alamos report LA-12116-T, (unpublished).

(Lo54) F. E. Low, Phys. Rev. 96, 1428 (1954).

(MC74) L.C. Maximon and J.S. O'Connell, Phys. Lett. 48B, 399 (1974).

(Me88) Werner Meyer, "Polarized Target Physics at the Bonn Electron Accelerators", BONN-IR-88-60.

(Mu93) L. Murphy, from recent measurements by the DAPHNE collaboration, private communication.

(Os68) H. Osborn, Phys. Rev. 176, 1523 (1968).

(Pe81) V. A. Petrunkin, Sov. J. Part. Nucl. 12, 278 (1981).

(SWK94) A.M. Sandorfi, C.S. Whisnant and M. Khandaker, Phys. Rev. D50 (in press).

(ST93) J. Soffer and O. Teryaev, Phys. Rev. Lett. 70, 3373 (1993).

(Wa84) Wabe, Heeringa, p. 129 in "Proceedings of the $4^{\text {th }}$ international Workshop on Polarized Target Materials and Techniques", Bad Honef, Germany, September 3-6, 1984, W. Meyer, editor.

(WA92) R. L. Workman and R. A. Arndt, Phys. Rev. D45, 1789 (1992).

(WA93) R. L. Workman and R. A. Arndt, private comm.; the Scattering Analysis Interactive Dial-in (SAID) program, available by TELNET to VTINTE.

(Wi88) S. L. Wilson, R. Hofstadter, E. B. Hughes, Y. C. Lin, R. Parks, M. W. Ritter, R. D. Bolton, J. D. Bowman, M. D. Cooper, J. S. Frank, A. L. Hallin, P. Heusi, C. M. Hoffman, G. E. Hogan, F. G. Mariam, H. S. Matis, R. E. Mischke, D. E. Nagle, L. E. Piilonen, V. D. Sandberg, G. H. Sanders, U, Sennhauser, R. Werbeck, R. A. Williams, D. P. Grosnick, S. C. Wright and J. McDonough, Nucl. Inst. Meth. A264, 263 (1988). 


\section{Appendix: Technical Review, June 7, 1994}

On June 7, 1994 a Technical Review was held at Brookhaven National Laboratory. The entire project was presented, although the main focus centered on the key piece of new technology, the SPHICE target. The review panel consisted of recognized experts in polarized target technology, namely Drs. D.G. Crabb from University of Virginia, P.P.J. Delheij from TRIUMF and L. Knutson from the University of Wisconsin. Their report, together with its recommendations, follows. BNL action on their recommendations is outlined in the next section.

The planned target presented to the review committee would have achieved inbeam relaxation times of about 5 days for hydrogen and 15 days for deuterium, with holding conditions of $1.3 \mathrm{~T}$ and $1.5^{\circ} \mathrm{K}$. Technical advances since the time of the June review have improved the expected target performance considerably. These developments are reflected in the discussions of the SPHICE target in this report, while the comments of the Technical Review Panel refer only to the target parameters assumed at the time of the June, 1994 review.

\section{Report of the Technical Review Panel on SPHICE - The HD Polarized Target for LEGS}

D.G. Crabb, P.P.J. Delheij and L. Knutson

(June 15, 1994)

Technical capabilities have advanced to a point where an experiment at LEGS on the structure of the nucleon is both feasible and timely. There are several factors that make such an experiment attractive. The LEGS facility is available on a routine basis, and the group has a considerable amount of experience in producing and using polarized gammaray beams. Many components of the detector package are already on hand. Finally, recent developments have improved the prospects for producing large samples of polarized HD. The proposed target has a number of unique features which match well to the experimental requirements. These include open geometry which allows large angular coverage and the fact that a large fraction of the nucleons in the target are polarized.

The committee believes that the proposed experiment is fundamentally sound. However, we feel that the group has not yet defined adequately a set of realistic goals for the experiment. What is the desired accuracy for the quantities that enter into the sum rules? What level of accuracy is needed for the absolute target polarization? What is the required statistical accuracy, and how does this translate into requirements on target polarization and on the number of days of beam time required with polarized hydrogen and polarized deuterium? These questions impact the design of the experiment and therefore must be addressed. 
(1) Technical Assessments

The proposed polarized target requires magnetic fields of $15 \mathrm{~T}$ to $17 \mathrm{~T}$ and temperatures near $15 \mathrm{mK}$ in a volume large enough to hold four $20 \mathrm{cc}$ samples. The conditions must be maintained for periods of 40-50 days to achieve reasonable polarization. The requirements on field, temperature and reliability approach the state of the art but should be achievable.

The relaxation times need to be short at $17 \mathrm{~T}$ and $15 \mathrm{mK}$, and long at $1.5 \mathrm{~K}$ and $1.3 \mathrm{~T}$. Measured relaxation times appear to be compatible with the experimental requirements, and suggest that one can maintain a moderately high level of polarization for periods of up to one week. Radiation damage should not be a serious problem.

The process of transferring samples from the dilution refrigerator to the storage and target dewars is well understood. While the process is straightforward, the amount of specialized equipment required is significant, and one can anticipate that substantial manpower will be required for the design and maintenance of this equipment.

\section{(2) Target Polarization}

The maximum polarizations achieved to date are $60 \%$ for protons and $38 \%$ for deuterons. The volume of the sample in this test was approximately $1 / 3 \mathrm{cc}$ in volume and contained large amounts of copper. The polarizing field was $12 \mathrm{~T}$ and the dilution refrigerator was operated for about 6 days. Adiabatic fast passage was used to increase the deuteron polarization from $28 \%$ (obtained by relaxation via para-D2 impurities) to $38 \%$. From here one needs to extrapolate to $20 \mathrm{cc}, 17 \mathrm{~T}$, cooling with imbedded aluminum wire, 40-50 day operation, and deuteron polarization via fast passage. Based on achieved performance and measured relaxation times, one can expect an initial deuteron polarization of around $46 \%$. Higher initial polarizations are possible with improvements in temperature or in the forbidden transition efficiency.

We believe that it is realistic to expect initial deuteron polarizations of $50 \%$, with the possibility of some small improvement. While we have identified no specific area of concern, there is always some risk associated with extrapolation into new regimes. We should also be aware of the fact that solid HD targets have never been used in nuclear physics experiments before. This requires a level of reliability significantly above that needed for test measurements. It is also important to remember that the experiment requires a very large number of samples. (The proposal mentions a running time of 10 months. At 6 days per sample, this requires the polarizing of about 50 samples. Production time is about 40-50 days for 4 samples, corresponding to over 1.5 years of dilution refrigerator operation). Maintaining average initial polarizations of $50 \%$ will clearly require exceptional reliability of the magnet/dilution refrigerator system, as well as error-free transfer and storage.

It is planned to use target samples for periods of about 6 days. This is roughly the polarization decay time, and therefore the average polarization will be about $32 \%$, 
assuming $50 \%$ initial polarization. It is not yet clear what the impact of lower polarization would be on the quality of the experiment.

Another serious issue concerns the determination of the absolute target polarization. It appears that $3 \%$ absolute accuracy would be desirable. This is essentially state-of-the-art. The committee feels that the group does not yet have a clear plan of how to achieve this level of accuracy in the in-beam dewar. It seems clear that some sort of online polarization monitor is essential. The goal of $1 \%$ absolute accuracy is probably not practical.

\section{(3) Manpower}

The committee is concerned about manpower. The number of post-docs and students seems adequate, but the number of senior collaborators with relevant experience concerning polarized target issues is not sufficient. There are a very large number of important jobs to be done that require expertise in this area:

1. Specifying and ordering the dilution fridge.

2. Specifying and ordering the polarizing magnet.

3. Testing and bringing both into operation.

4. Research and development of viable targets.

5. HD distillation.

6. Specification, design, production and testing of in-beam magnet.

7. Transportation dewars - procurement and testing.

8. Specification, design, production and testing of in-beam NMR.

It appears that Honig is being counted on to do a large number of these tasks. Honig has infinite enthusiasm, nearly infinite energy but not infinite time. The situation is far from ideal. Honig is not a nuclear physicist, and we believe that there is a risk that interesting condensed matter physics problems will be pursued at the expense of practical target development. It is important to identify someone (preferably from BNL) with sufficient seniority and expertise to assist with maintaining the target construction schedule of 2 to 3 years. This will also ensure a smooth transition for operation at LEGS.

\section{(4) Recommendations}

We recommend proceeding with the procurement of the dilution refrigerator together with the matching $17 \mathrm{~T}$ polarizing magnet. In procurement of large dewars and cryostats it is important to request from the manufacturer applicable codes to which the device was built. This should include such items as weld call outs, etc. This will help for lab cryogenic safety review. The cost of these items seem to be well estimated.

We suggest that BNL exercise care in selection of the vendor for the dilution refrigerator/magnet system. At the minimum the laboratory should require prospective vendors to provide lists of customers who have purchased similar systems. If the 
proposed device differs substantially from standard designs, the extent of the modifications should also be made clear.

We recommend that $\mathrm{BNL}$ allocate funds (presumably around $\$ 20 \mathrm{k}$ ) for NMR systems and/or magnet modifications to allow for measuring the target polarization in the gamma-ray beam.

Finally, we recommend that BNL identify a person or persons (preferably senior and preferably with polarized target experience) to play a central role in the target development.

\section{(5) Budgets}

The costs of the large capital equipment items seems realistic. In addition to the $20 \mathrm{k}$ recommended for the in-beam NMR system, there needs to be an entry in the budget for the variable temperature $H D$ condensing refrigerator. It was suggested that ORSAY could provide this device, but this should be confirmed. The cost is about $\$ 20 \mathrm{k}$.

Finally we note that, for the most part, the budget contains only the large capital items and the salaries of some of the central people. It should be clear that the project will have a substantial impact on various operating budgets.

\section{BNL Action on Recommendations of the Iune 7, 1994 Technical Review}

The specific recommendations of the June 7, 1994 Technical Review are listed here, together with the corresponding responses from BNL.

\section{(1) Recommendation:}

"We recommend proceeding with the procurement of the dilution refrigerator together with the matching $17 \mathrm{~T}$ polarizing magnet. In procurement of large dewars and cryostats it is important to request from the manufacturer applicable codes to which the device was built. This should include such items as weld call outs, etc. This will help for lab cryogenic safety review. The cost of these items seem to be well estimated.

We suggest that BNL exercise care in selection of the vendor for the dilution refrigerator/magnet system. At the minimum, the laboratory should require prospective vendors to provide lists of customers who have purchased similar systems. If the proposed device differs substantially from standard designs, the extent of the modifications should also be made clear."

Action:

Following a competitive bid solicitation, an order has been placed with Oxford Instruments Inc. for a Model 1000 dilution refrigerator system, integrated with a 15-17 T superconducting magnet. Performance testing at their facilities is scheduled for June, 1995, and final delivery is expected by mid-August, 1995.

Oxford supplied names of recent customers for similar such systems and BNL held extensive discussions with one of these. The refrigerator for BNL will be constructed along 
a proven existing technology. The only significantly new development over older models is the complete computer control of the system. This has been implemented with National Instruments LABVIEW, a widely used commercial software product. Oxford has already sold six such completely automated systems.

Oxford has agreed to conform to all DOE/BNL QA standards. In particular, all pressure and vacuum welds will be radiographed and certified to meet the ISO-9000 code.

(2) Recommendation:

"We recommend that BNL allocate funds (presumably around $\$ 20 \mathrm{k}$ ) for NMR systems and/or magnet modifications to allow for measuring the target polarization in the gamma-ray beam."

Action:

Design of in-beam polarization monitoring will begin in FY 1995. The costs of the associated equipment will be spread out over FY 1995 and FY 1996.

(3) Recommendation:

"Finally, we recommend that BNL identify a person or persons (preferably senior and preferably with polarized target experience) to play a central role in the target development.

Action:

Funds have been allocated, starting in FY 1995, for the addition of a physicist with extensive experience in both Nuclear Physics and ultra-low-temperature cryogenics. The search for a qualified candidate has already begun. 